\title{
Generalized dice similarity measures for complex q-Rung Orthopair fuzzy sets and its application
}

\author{
Harish Garg $^{1}$ (I) $\cdot$ Zeeshan Ali $^{2} \cdot$ Tahir Mahmood $^{2}$
}

Received: 3 January 2020 / Accepted: 15 September 2020 / Published online: 13 November 2020

(c) The Author(s) 2020

\begin{abstract}
Complex q-rung orthopair fuzzy set (Cq-ROFS) is an extension of Complex fuzzy set, intuitionistic fuzzy set, Pythagorean fuzzy set, to cope with complicated and inconsistence information in the environment of fuzzy set theory with a wider domain. In Cq-ROFS, each attribute is characterized by the degree of membership and non-membership degree over the unit-disc of the complex plan. Keeping the advantages of Cq-ROFSs, in this manuscript, we present a concept of the dice similarity and generalized dice similarity measures between the pairs of the sets. The basic axioms and properties are also stated. Further, we extend the proposed measures to weighted dice similarity measures and investigated their properties. The certain properties and the special cases of the proposed work are also derived. The applicability of the proposed measures is demonstrated with some numerical examples related to medical diagnoses and pattern recognition. The superiority and advantages of the measures over the existing ones are also illustrated with certain numerical examples.
\end{abstract}

Keywords Complex q-Rung Orthopair fuzzy Sets · Dice similarity Measures · Generalized dice similarity measures · Medical diagnosis

\section{Introduction}

Decision making process includes the examination of a limited arrangement of options and positioning them as far as the fact that they are so trustworthy to decision-maker(s) when all the rules are thought of at the same time. In this procedure, the rating estimations of every option incorporate both exact information and specialists' subjective data. However, generally, the information which is collected from the various data sources is limited in nature and involves high range of uncertainties. To cope with such uncertainties, a theory of fuzzy set (FS), developed by Zadeh [1] provides an important tool to deal with uncertain and unpredictable information in the environment of real life issues. In FS, each element consists of the membership function, whose range is $[0,1]$. Since their appearance, a lot of attentions have been made by the

Harish Garg

harishg58iitr@gmail.com

1 School of Mathematics, Thapar Institute of Engineering and Technology, Deemed University, Patiala 147004, Punjab, India

2 Department of Mathematics and Statistics, International Islamic University, Islamabad, Pakistan researchers and applied them in the different fields such as aggregation operators [2], medical diagnosis [3] etc. The theory of FS is widely used but their scope is limited in nature due to the consideration of only membership degree.

Atanassove's-intuitionistic fuzzy set (A-IFS) [4] is a generalization of FS, which described the truth degree and the falsity degree with a condition that the sum of truth degree and falsity degree is less than or equal to one. A-IFS can help to deal with complicated and difficult information. After its appearance, the A-IFS is received more attentions in the environment of pattern recognition [5, 6], medical diagnosis [7, 8], aggregation operators [9], distance and similarity measures $[10,11]$. As the A-IFS is much widely used by the researchers to solve the decision making problems and to express the uncertainties in the data. But it is still observed that the feasible region of A-IFS is a triangular region and is limited in access. For example, when a decision maker provides a source of information such as $(0.7,0.5)$, where the values of membership and non-membership grades with a condition such that $0.7+0.5=1.2>1$, and hence under such cases, the IFS cannot cope effectively.

To address such situation more clearly and increase the searching space, Yager [12] initiated the idea of Pythagorean fuzzy set (PFS) as a generalization of A-IFS, characterized

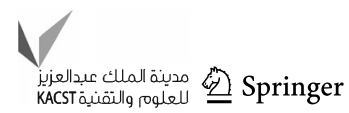



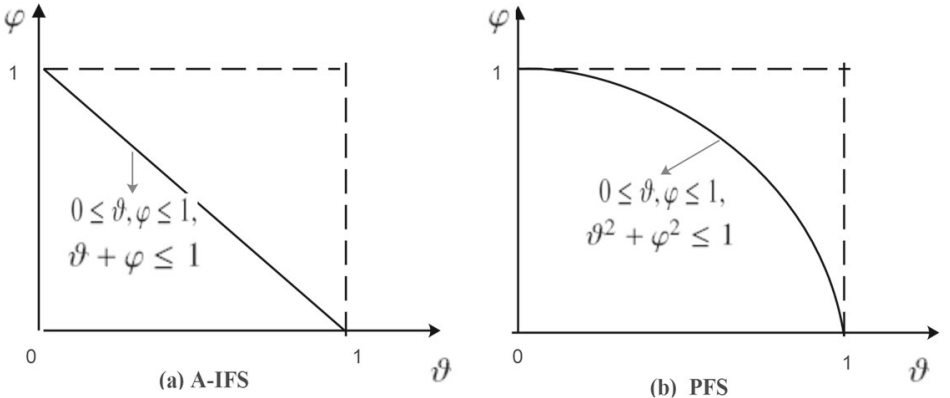

(b) PFS

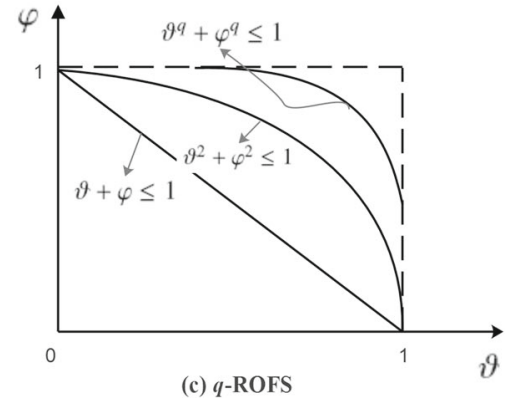

(c) $q$-ROFS

Fig. 1 Geometric interpretation of A-IFS, PFS, and q-ROFS

by membership degree and non-membership degree with a condition such that the sum of the square of membership degree and the square of the non-membership degree is less than or equal to 1 . From their structure, it is considered that the searching space of this set is wider than the FS, A-IFS. For instance, a pair $(0.7,0.5)$ is well handled with the PFS as $0.7^{2}+0.5^{2}=0.74 \leq 1$, the PFS cope effectively. To study the influence of the PFS on to the decision making process, several researchers have put forwards the different kinds of the algorithms. For instance, Garg [13] studied the correlation coefficient based on the pairs of the PFSs. Peng and Yang [14] discussed some different kinds of the basic results of PFSs. Zhang and $\mathrm{Xu}$ [15] presented a TOPSIS ("Technique of Order Preference Similarity to the Ideal Solution") method under the PFS environment to solve the decision making problems. Garg [16, 17] initiated the weighted aggregation operators for the PFS. Peng and Garg [18] presented a multiparametric similarity measures for PFS. Wang and Li [19] presented Pythagorean fuzzy interactive power Bonferroni mean operators to solve the problems. Garg [20, 21] presented new kinds of aggregation operations based on the concept of neutrality operations for PFSs and hence presented algorithms to solve the multiple attribute group decision making problems. A different approach to solve the problem under the PFS is reviewed by Peng and Selvachandran [22].

Yager [23] presented the concept of more generalized fuzzy set named as q-rung orthopair fuzzy set (q-ROFS), which is an extension of the existing A-IFS and PFS. In this set, a parameter called as rung "q" is a flexible parameter which can describe the strengthen of the set by assigning it to the membership degrees of the set. The constraint of qROFS is that the sum of the q-power of membership degree and the q-power of the non-membership degree is less than or equal to 1 . To better understand it, consider a pair of the membership degrees as $(0.9,0.8)$, then clearly seen that $0.9+0.8 \geq 1, \quad 0.9^{2}+0.8^{2} \geq 1$ and hence A-IFS and PFS are not capable to handle such situation. On the other hand, we can see that $0.9^{q}+0.8^{q} \leq 1$ for $q=5$. Thus, the q-ROFS cope effectively. Under the q-ROFS, Liu and Wang [24] presented an aggregation operator based on q-ROFS. Wei et al.
[25] proposed Heronian mean operators based on q-ROFS. Liu and Wang [26] initiated Archimedean Bonferroni mean operators based on q-ROFS. Garg and Chen [27] discussed the concept of neutrality operations and it's based operatoes under the q-ROFS environment. Liu and Liu [28] initiated Bonferroni mean operators based on q-ROFS. Peng et al. [29] presented exponential operators for q-ROFS to solve the decision-making problems. Xing et al. [30] presented some point operators for the q-rung orthopair fuzzy sets and its based point weighted aggregation operators for solving the decision making problems. Recently, Garg [31] presented a novel trigonometric operation law and their aggregation operators to solve the problems. The geometrical interpretation of q-ROFS and their existing methods are shown in Fig. 1.

From the above investigation, it is studied that all the studies mentioned above are based on the pairs of the real numbers. To address the problem more clearly, Ramot et al. [32] presented the notion of complex fuzzy set (CFS), which extend the range of the membership degrees from real set to the unit disc in a complex plane. However, a concept of complex fuzzy logic was discussed by Ramot et al. [33]. In 2012, Alkouri and Salleh [34] extended the concept of CFS to complex intuitionistic fuzzy set (CIFS) by considering the complex degree of non-membership grades. The CIFS contains two dimensions information in a single set. Kumar and Bajaj [35], founded on complex intuitionistic fuzzy soft sets with distance measures and entropies. Garg and Rani [36, 37] presented a robust correlation coefficient and aggregation operators for a pair of CIFS. Rani and Garg [38] presented a distance measures between the pairs of the CIFSs while Rani and Garg [39] initiated the idea of the power aggregation operators for CIFS to solve the decision making problems. Recently, Garg and Rani [40] initiated the idea of complex interval-valued intuitionistic fuzzy sets (CIVFSs) and their aggregation operators. Ullah et al. [41] combined the idea of PFS with CFS is to proposed complex Pythagorean fuzzy set (CPFS) to handle uncertainty. Akram et al. [42] presented a TOPSIS and ELECTRE method under CPFS environment to solve group decision making problems. Liu et al. [43] and Garg et al. [44] explored the novel approach of com- 
plex q-rung orthopair fuzzy set (Cq-ROFS), as a mixture of q-rung orthopair fuzzy set and complex fuzzy set to cope with awkward and complicated information in realistic decision theory. The Cq-ROFS contains grade of supporting and grade of supporting against in the form of polar co-ordinates belonging to unit disc in a complex plane. The condition of Cq-ROFS is that the sum of q-powers of the real parts (also for imaginary parts) of the truth grade and falsity grade is not exceeded from unit interval. A Cq-ROFS is a progressively summed up augmentation of the current speculations, for example, FSs, A-IFSs, PFSs, q-ROFSs, CFSs, CIFSs, CPFSs, and it can considerably more information to communicate the data.

Subsequently, the advantages of Cq-ROFS, have driven us to think about the accompanying primary goals for this paper. Our proposed work has four important parts. First is to present the concept of the dice similarity measures and generalized dice similarity for Cq-ROFS. The similarity measure is one of the information measures which is used to compute the degree of similarity between the given set. In other words, it quantifies the closeness degree between the pairs of the sets. The special cases of the investigated measures are discussed with the help of some Remark. Further, we extend them the presented measures to the weighted measures. To address their applicability, some suitable numerical examples are taken from the field of the pattern recognition and medical diagnosis. The efficiency and effectiveness of the proposed measure are also demonstrated with a numerical example.

At the nutshells, the main objective of the paper is of summarized as follow:

1. To represent the preference of the decision makers in terms of complex q-rung orthopair fuzzy (Cq-ROF) numbers.

2. To present some new dice similarity measures to find the degree of similarity between the pairs of Cq-ROFS.

3. To examine some numerical example to demonstrate the working of the proposed measures.

4. The effectiveness of the proposed measures is discussed with some counter-intuitive cases.

The rest of the manuscript is set out as follows. In section "Preliminaries", we reviewed the basic definitions of PFS, q-ROFS, Cq-ROFS. In section "Dice similarity measures for complex q-Rung Orthopair fuzzy sets", a concept of dice and generalized dice similarity for $\mathrm{Cq}$-ROFS are stated and investigated their properties. The special cases of the investigated measures are discussed with the help of some Remark. In section "Illustrative examples", we illustrate the proposed measures with some numerical examples related to pattern recognition and medical diagnosis. Some examples are also provided to demonstrate the efficiency and effectiveness of the measures. Finally, the conclusion is summarized in section "Conclusion".

\section{Preliminaries}

In this section, we discuss basic notions of PFSs, CPFSs, q-ROFSs and DSMs.

Definition 1 [12] A PFS $A$ is denoted and given by:

$A=\left\{\left(\alpha_{A}^{\prime}(x), \beta_{A}^{\prime}(x)\right): x \in X\right\}$

where $\alpha_{A}^{\prime}, \quad \beta_{A}^{\prime}: X \rightarrow[0,1]$ represent the degree of membership and the degree of non-membership, with a condition $0 \leq \alpha_{A}^{\prime 2}+\beta_{A}^{\prime 2} \leq 1$. The hesitancy degree is follow as: $\theta_{A}=$ $\left(1-\alpha_{A}^{\prime 2}-\beta_{A}^{\prime 2}\right)^{\frac{1}{2}}$.

Definition 2 [41] A CPFS $A$ is denoted and given by:

$A=\left\{\left(\alpha_{A}^{\prime}(x), \beta_{A}^{\prime}(x)\right): x \in X\right\}$

where $\alpha_{A}^{\prime}=\alpha_{A} e^{i 2 \pi\left(\delta_{\alpha_{A}}\right)}$ and $\beta_{A}^{\prime}=\beta_{A} e^{i 2 \pi\left(\delta_{\beta_{A}}\right)}$ are represented the degree of complex-valued membership and the degree of complex-valued non-membership, with a conditions $0 \leq \alpha_{A}^{2}+\beta_{A}^{2} \leq 1$ and $0 \leq \delta_{\alpha_{A}}^{2}+$ $\delta_{\beta_{A}}^{2} \leq 1$. The hesitancy degree is follow as: $\theta_{A}=$ $\left(1-\alpha_{A}^{2}-\beta_{A}^{2}\right)^{\frac{1}{2}} e^{\mathrm{i} 2 \pi\left(1-\delta_{\alpha_{A}}^{2}-\delta_{\beta_{A}}^{2}\right)^{\frac{1}{2}}}$.

Definition 3 [23] A q-ROFS $A$ is denoted and given by: 
$A=\left\{\left(\alpha_{A}^{\prime}(x), \beta_{A}^{\prime}(x)\right): x \in X\right\}$

where $\alpha_{A}^{\prime}, \beta_{A}^{\prime}: X \rightarrow[0,1]$ represent the degree of membership and the degree of non-membership, with a condition $0 \leq \alpha_{A}^{\prime}{ }^{q}+\beta_{A}^{\prime q} \leq 1, q \geq 1$. The hesitancy degree is follow as: $\theta_{A}=\left(1-\alpha_{A}^{\prime q}-\beta_{A}^{\prime q}\right)^{\frac{1}{q}}$.

Definition 4 [45] Considered the two vectors $X=$ $\left(x_{1}, x_{2}, \ldots, x_{n}\right)$ and $Y=\left(y_{1}, y_{2}, \ldots, y_{n}\right)$, then the DSM is denoted and defined by:

$d(X, Y)=\frac{2 X \cdot Y}{\|X\|_{2}^{2}+\|Y\|_{2}^{2}}=\frac{2 \sum_{i=1}^{n} x_{i} y_{i}}{\sum_{i=1}^{n} x_{i}^{2}+\sum_{i=1}^{n} y_{i}^{2}}$

where $X \cdot Y=\sum_{i=1}^{n} x_{i} y_{i}$ represents the inner products and $X_{2}^{2}=\sum_{i=1}^{n} x_{i}^{2}, Y_{2}^{2}=\sum_{i=1}^{n} y_{i}^{2}$ represents the Euclidean norm of vectors $X$ and $Y$. If $x_{i}=y_{i}=0$, so the DSM is undefined.

Definition $5[43,44]$ A CPFS $A$ is denoted and given by:

$A=\left\{\left(\alpha_{A}^{\prime}(x), \beta_{A}^{\prime}(x)\right): x \in X\right\}$

where $\alpha_{A}^{\prime}=\alpha_{A} e^{i 2 \pi\left(\delta_{\alpha_{A}}\right)}$ and $\beta_{A}^{\prime}=\beta_{A} e^{i 2 \pi\left(\delta_{\beta_{A}}\right)}$ are represented the degree of complex-valued membership and the degree of complex-valued non-membership, with a conditions $0 \leq \alpha_{A}^{q}+\beta_{A}^{q} \leq 1$ and $0 \leq$ $\delta_{\alpha_{A}}^{q}+\delta_{\beta_{A}}^{q} \leq 1$. The hesitancy degree is defined as: $\theta_{A}=\left(1-\alpha_{A}^{q}-\beta_{A}^{q}\right)^{\frac{1}{q}} e^{\mathrm{i} 2 \pi\left(1-\delta_{\alpha_{A}}^{q}-\delta_{\beta_{A}}^{q}\right)^{\frac{1}{q}}}$. The complex q-rung orthopair fuzzy number is represented by $A=$ $\left(\alpha_{A}\left(x_{i}\right) e^{i 2 \pi\left(\delta_{\alpha_{A}}\left(x_{i}\right)\right)}, \beta_{A}\left(x_{i}\right) e^{i 2 \pi\left(\delta_{\beta_{A}}\left(x_{i}\right)\right)}\right)$. The set of CqROFSs is denoted by $C q-R O F S(X)$.

Definition $6[43,44]$ Let $A, B \in C q-\operatorname{ROFS}(X)$, then the following are hold true

1. $A^{c}=\left(\beta_{A}\left(x_{i}\right) e^{i 2 \pi\left(\delta_{\beta_{A}}\left(x_{i}\right)\right)}, \alpha_{A}\left(x_{i}\right) e^{i 2 \pi\left(\delta_{\alpha_{A}}\left(x_{i}\right)\right)}\right)$;

2. $A \subseteq B$ if $\alpha_{A}\left(x_{i}\right) \leq \alpha_{B}\left(x_{i}\right), \delta_{\alpha_{A}}\left(x_{i}\right) \leq \delta_{\alpha_{B}}\left(x_{i}\right), \beta_{A}$ $\left(x_{i}\right) \geq \beta_{B}\left(x_{i}\right)$ and $\delta_{\beta_{A}}\left(x_{i}\right) \geq \delta_{\beta_{B}}\left(x_{i}\right)$;

3. $A=B$ iff $A \subseteq B$ and $A \supseteq B$.
Dice similarity measures for complex q-Rung Orthopair fuzzy sets

In this section, we propose the notion of dice similarity measure (DSM) and weighted DSM (WDSM) for Cq-ROFSs. The special cases of the proposed approaches are also discussed in detail. Throughout this article, the weight vector is given by: $\omega=\left\{\omega_{1}, \omega_{2}, \ldots, \omega_{n}\right\}, \omega_{i}>0$ with a condition $\sum_{i=1}^{n} \omega_{i}=1$.

\section{Dice and weighted dice similarity measures}

Let $A=\left(\alpha_{A}\left(x_{i}\right) e^{i 2 \pi\left(\delta_{\alpha_{A}}\left(x_{i}\right)\right)}, \beta_{A}\left(x_{i}\right) e^{i 2 \pi\left(\delta_{\beta_{A}}\left(x_{i}\right)\right)}\right)$ be a CqROFS defined on a finite universal set $X$.

Definition 7 For two Cq-ROFS A and B, a DSM $d_{C q-R O F}^{1}$ $(A, B)$ for Cq-ROFSs is given by:

$$
\begin{aligned}
& d_{C q-R O F}^{1}(A, B) \\
& =\frac{1}{n} \sum_{i=1}^{n}\left(\begin{array}{c}
2\left(\begin{array}{c}
\alpha_{A}^{q}\left(x_{i}\right) \alpha_{B}^{q}\left(x_{i}\right)+\beta_{A}^{q}\left(x_{i}\right) \beta_{B}^{q}\left(x_{i}\right) \\
+\delta_{\alpha_{A}}^{q}\left(x_{i}\right) \delta_{\alpha_{B}}^{q}\left(x_{i}\right)+\delta_{\beta_{A}}^{q}\left(x_{i}\right) \delta_{\beta_{B}}^{q}\left(x_{i}\right)
\end{array}\right) \\
\left(\begin{array}{l}
\left.\alpha_{A}^{2 q}\left(x_{i}\right)+\beta_{A}^{2 q}\left(x_{i}\right)+\delta_{\alpha_{A}}^{2 q}\left(x_{i}\right)+\delta_{\beta_{A}}^{2 q}\left(x_{i}\right)\right) \\
+\left(\alpha_{B}^{2 q}\left(x_{i}\right)+\beta_{B}^{2 q}\left(x_{i}\right)+\delta_{\alpha_{B}}^{2 q}\left(x_{i}\right)+\delta_{\beta_{B}}^{2 q}\left(x_{i}\right)\right)
\end{array}\right)
\end{array}\right)
\end{aligned}
$$

which satisfy following conditions:

1. $0 \leq d_{C q-R O F}^{1}(A, B) \leq 1$

2. $d_{C q-R O F}^{1}(A, B)=d_{C q-R O F}^{1}(B, A)$

3. $d_{C q-R O F}^{1}(A, B)=1$ if $A=B$.

Proof Let $A=\left(\alpha_{A}\left(x_{i}\right) e^{i 2 \pi\left(\delta_{\alpha_{A}}\left(x_{i}\right)\right)}, \beta_{A}\left(x_{i}\right) e^{i 2 \pi\left(\delta_{\beta_{A}}\left(x_{i}\right)\right)}\right)$ and $B=\left(\alpha_{B}\left(x_{i}\right) e^{i 2 \pi\left(\delta_{\alpha_{B}}\left(x_{i}\right)\right)}, \beta_{B}\left(x_{i}\right) e^{i 2 \pi\left(\delta_{\beta_{B}}\left(x_{i}\right)\right)}\right)$ are two Cq-ROFSs. We will consider the Eq. (6), and prove the follow conditions:

1. Let 
$d_{\mathrm{Cq}-\mathrm{ROF}}^{1}(A, B)$

$=\frac{1}{n} \sum_{i=1}^{n}\left(\begin{array}{c}2\left(\begin{array}{c}\alpha_{A}^{q}\left(x_{i}\right) \alpha_{B}^{q}\left(x_{i}\right)+\beta_{A}^{q}\left(x_{i}\right) \beta_{B}^{q}\left(x_{i}\right) \\ +\delta_{\alpha_{A}}^{q}\left(x_{i}\right) \delta_{\alpha_{B}}^{q}\left(x_{i}\right)+\delta_{\beta_{A}}^{q}\left(x_{i}\right) \delta_{\beta_{B}}^{q}\left(x_{i}\right)\end{array}\right) \\ \left(\begin{array}{c}\left.\alpha_{A}^{2 q}\left(x_{i}\right)+\beta_{A}^{2 q}\left(x_{i}\right)+\delta_{\alpha_{A}}^{2 q}\left(x_{i}\right)+\delta_{\beta_{A}}^{2 q}\left(x_{i}\right)\right) \\ +\left(\alpha_{B}^{2 q}\left(x_{i}\right)+\beta_{B}^{2 q}\left(x_{i}\right)+\delta_{\alpha_{B}}^{2 q}\left(x_{i}\right)+\delta_{\beta_{B}}^{2 q}\left(x_{i}\right)\right)\end{array}\right)\end{array}\right)$

It is clear that $d_{\mathrm{Cq}-\mathrm{ROF}}^{1}(A, B) \geq 0$, and

$$
\begin{gathered}
\left(\begin{array}{l}
\left(\alpha_{A}^{2 q}\left(x_{i}\right)+\beta_{A}^{2 q}\left(x_{i}\right)+\delta_{\alpha_{A}}^{2 q}\left(x_{i}\right)+\delta_{\beta_{A}}^{2 q}\left(x_{i}\right)\right) \\
\left(+\alpha_{B}^{2 q}\left(x_{i}\right)+\beta_{B}^{2 q}\left(x_{i}\right)+\delta_{\alpha_{B}}^{2 q}\left(x_{i}\right)+\delta_{\beta_{B}}^{2 q}\left(x_{i}\right)\right)
\end{array}\right) \\
\quad \geq 2\left(\begin{array}{c}
\alpha_{A}^{q}\left(x_{i}\right) \alpha_{B}^{q}\left(x_{i}\right)+\beta_{A}^{q}\left(x_{i}\right) \beta_{B}^{q}\left(x_{i}\right) \\
+\delta_{\alpha_{A}}^{q}\left(x_{i}\right) \delta_{\alpha_{B}}^{q}\left(x_{i}\right)+\delta_{\beta_{A}}^{q}\left(x_{i}\right) \delta_{\beta_{B}}^{q}\left(x_{i}\right)
\end{array}\right)
\end{gathered}
$$

Thus, according to the inequality $a^{2 q}+b^{2 q} \geq 2 a^{q} b^{q}$, we can get, $0 \leq d_{\mathrm{Cq}-\mathrm{ROF}}^{1}\left(A_{i}, B_{i}\right) \leq 1$, so from Eq. (6), we get $0 \leq d_{\mathrm{Cq}-\mathrm{ROF}}^{1}(A, B) \leq 1$.

2. The Eq. (6), is easily verified the condition 2 .

3. If $A=B$, i.e. $\alpha_{A}^{q}\left(x_{i}\right)=\alpha_{B}^{q}\left(x_{i}\right), \beta_{A}^{q}\left(x_{i}\right)=\beta_{B}^{q}\left(x_{i}\right), \delta_{\alpha_{A}}^{q}$ 4. $\left(x_{i}\right)=\delta_{\alpha_{B}}^{q}\left(x_{i}\right)$ and $\delta_{\beta_{A}}^{q}\left(x_{i}\right)=\delta_{\beta_{B}}^{q}\left(x_{i}\right)$, we have

$$
\begin{array}{r}
=\frac{1}{n} \sum_{i=1}^{n} \frac{2\left(\alpha_{A}^{q}\left(x_{i}\right) \alpha_{B}^{q}\left(x_{i}\right)+\beta_{A}^{q}\left(x_{i}\right) \beta_{B}^{q}\left(x_{i}\right)+\delta_{\alpha_{A}}^{q}\left(x_{i}\right) \delta_{\alpha_{B}}^{q}\left(x_{i}\right)+\delta_{\beta_{A}}^{q}\left(x_{i}\right) \delta_{\beta_{B}}^{q}\left(x_{i}\right)\right)}{\left(\alpha_{A}^{2 q}\left(x_{i}\right)+\beta_{A}^{2 q}\left(x_{i}\right)+\delta_{\alpha_{A}}^{2 q}\left(x_{i}\right)+\delta_{\beta_{A}}^{2 q}\left(x_{i}\right)\right)+\left(\alpha_{B}^{2 q}\left(x_{i}\right)+\beta_{B}^{2 q}\left(x_{i}\right)+\delta_{\alpha_{B}}^{2 q}\left(x_{i}\right)+\delta_{\beta_{B}}^{2 q}\left(x_{i}\right)\right)} \\
=\frac{1}{n} \sum_{i=1}^{n} \frac{2\left(\alpha_{A}^{q}\left(x_{i}\right) \alpha_{A}^{q}\left(x_{i}\right)+\beta_{A}^{q}\left(x_{i}\right) \beta_{A}^{q}\left(x_{i}\right)+\delta_{\alpha_{A}}^{q}\left(x_{i}\right) \delta_{\alpha_{A}}^{q}\left(x_{i}\right)+\delta_{\beta_{A}}^{q}\left(x_{i}\right) \delta_{\beta_{A}}^{q}\left(x_{i}\right)\right)}{\left(\alpha_{A}^{2 q}\left(x_{i}\right)+\beta_{A}^{2 q}\left(x_{i}\right)+\delta_{\alpha_{A}}^{2 q}\left(x_{i}\right)+\delta_{\beta_{A}}^{2 q}\left(x_{i}\right)\right)+\left(\alpha_{A}^{2 q}\left(x_{i}\right)+\beta_{A}^{2 q}\left(x_{i}\right)+\delta_{\alpha_{A}}^{2 q}\left(x_{i}\right)+\delta_{\beta_{A}}^{2 q}\left(x_{i}\right)\right)} \\
=\frac{1}{n} \sum_{i=1}^{n} \frac{2\left(\alpha_{A}^{2 q}\left(x_{i}\right)+\beta_{A}^{2 q}\left(x_{i}\right)+\delta_{\alpha_{A}}^{2 q}\left(x_{i}\right)+\delta_{\beta_{A}}^{2 q}\left(x_{i}\right)\right)}{2\left(\alpha_{A}^{2 q}\left(x_{i}\right)+\beta_{A}^{2 q}\left(x_{i}\right)+\delta_{\alpha_{A}}^{2 q}\left(x_{i}\right)+\delta_{\beta_{A}}^{2 q}\left(x_{i}\right)\right)} \\
=1
\end{array}
$$

which satisfy following conditions:

1. $0 \leq d_{\mathrm{Cq}-\mathrm{ROF}}^{\mathrm{w} 1}(A, B) \leq 1$

2. $d_{\mathrm{Cq}-\mathrm{ROF}}^{\mathrm{w} 1}(A, B)=d_{\mathrm{Cq}-\mathrm{ROF}}^{\mathrm{w} 1}(B, A)$

3. $d_{\mathrm{Cq}-\mathrm{ROF}}^{\mathrm{w} 1}(A, B)=1$ if $A=B$. 
Remark 1 If we take $\omega=\left(\frac{1}{n}, \frac{1}{n}, \ldots, \frac{1}{n}\right)^{T}$ then the WDSM for Cq-ROFS reduced to DSM for Cq-ROFS i.e. $d_{\mathrm{Cq}-\mathrm{ROF}}^{\mathrm{w} 1}$ $(A, B)=d_{\mathrm{Cq}-\mathrm{ROF}}^{1}(A, B)$.

Remark 2 The dice distance measure (DDM) and weighted dice distance measure (WDDM) for Cq-ROFSs are stated as follow:

$d d_{\mathrm{Cq}-\mathrm{ROF}}^{1}(A, B)=1-d_{\mathrm{Cq}-\mathrm{ROF}}^{1}(A, B)$

$d d_{\mathrm{Cq}-\mathrm{ROF}}^{\mathrm{w} 1}(A, B)=1-d_{\mathrm{Cq}-\mathrm{ROF}}^{\mathrm{w} 1}(A, B)$

Definition 9 A DSM $d_{\mathrm{Cq}-\mathrm{ROF}}^{2}(A, B)$ for Cq-ROFSs is given by:

$d_{\mathrm{Cq}-\mathrm{ROF}}^{2}(A, B)=\frac{1}{n} \sum_{i=1}^{n}\left(\frac{2\left(\begin{array}{c}\alpha_{A}^{q}\left(x_{i}\right) \alpha_{B}^{q}\left(x_{i}\right)+\beta_{A}^{q}\left(x_{i}\right) \beta_{B}^{q}\left(x_{i}\right)+\theta_{A}^{q}\left(x_{i}\right) \theta_{B}^{q}\left(x_{i}\right) \\ +\delta_{\alpha_{A}}^{q}\left(x_{i}\right) \delta_{\alpha_{B}}^{q}\left(x_{i}\right)+\delta_{\beta_{A}}^{q}\left(x_{i}\right) \delta_{\beta_{B}}^{q}\left(x_{i}\right)+\delta_{\theta_{A}}^{q}\left(x_{i}\right) \delta_{\theta_{B}}^{q}\left(x_{i}\right)\end{array}\right)}{\left(\begin{array}{c}\alpha_{A}^{2 q}\left(x_{i}\right)+\beta_{A}^{2 q}\left(x_{i}\right)+\theta_{A}^{2 q}\left(x_{i}\right) \\ +\delta_{\alpha_{A}}^{2 q}\left(x_{i}\right)+\delta_{\beta_{A}}^{2 q}\left(x_{i}\right)+\delta_{\theta_{A}}^{2 q}\left(x_{i}\right)\end{array}\right)+\left(\begin{array}{c}\alpha_{B}^{2 q}\left(x_{i}\right)+\beta_{B}^{2 q}\left(x_{i}\right)+\theta_{B}^{2 q}\left(x_{i}\right) \\ +\delta_{\alpha_{B}}^{2 q}\left(x_{i}\right)+\delta_{\beta_{B}}^{2 q}\left(x_{i}\right)+\delta_{\theta_{B}}^{2 q}\left(x_{i}\right)\end{array}\right)}\right)$
Remark 3 If we take $\omega=\left(\frac{1}{n}, \frac{1}{n}, \ldots, \frac{1}{n}\right)^{T}$ then the WDSM for Cq-ROFS reduced to DSM for Cq-ROFS i.e. $d_{\mathrm{Cq}-\mathrm{ROF}}^{\mathrm{w} 2}$ $(A, B)=d_{\mathrm{Cq}-\mathrm{ROF}}^{2}(A, B)$.

Remark 4 The DDM and WDDM for Cq-ROFSs are defined as:

$d d_{\mathrm{Cq}-\mathrm{ROF}}^{2}(A, B)=1-d_{\mathrm{Cq}-\mathrm{ROF}}^{2}(A, B)$

$d d_{\mathrm{Cq}-\mathrm{ROF}}^{\mathrm{w} 2}(A, B)=1-d_{\mathrm{Cq}-\mathrm{ROF}}^{\mathrm{w} 2}(A, B)$

Definition 11 A DSM $d_{\mathrm{Cq}-\mathrm{ROF}}^{3}(A, B)$ for Cq-ROFSs is given by:

which satisfy following conditions:

1. $0 \leq d_{\mathrm{Cq}-\mathrm{ROF}}^{2}(A, B) \leq 1$

2. $d_{\mathrm{Cq}-\mathrm{ROF}}^{2}(A, B)=d_{\mathrm{Cq}-\mathrm{ROF}}^{2}(B, A)$

3. $d_{\mathrm{Cq}-\mathrm{ROF}}^{2}(A, B)=1$ if $A=B$.

Definition 10 A WDSM $d_{\mathrm{Cq}-\mathrm{ROF}}^{\mathrm{w} 2}(A, B)$ for Cq-ROFSs is given by:

$$
\begin{aligned}
& d_{\mathrm{Cq}-\mathrm{ROF}}^{3}(A, B) \\
& =\left(\begin{array}{c}
\sum_{i=1}^{n} 2\left(\begin{array}{c}
\alpha_{A}^{q}\left(x_{i}\right) \alpha_{B}^{q}\left(x_{i}\right)+\beta_{A}^{q}\left(x_{i}\right) \beta_{B}^{q}\left(x_{i}\right) \\
+\delta_{\alpha_{A}}^{q}\left(x_{i}\right) \delta_{\alpha_{B}}^{q}\left(x_{i}\right)+\delta_{\beta_{A}}^{q}\left(x_{i}\right) \delta_{\beta_{B}}^{q}\left(x_{i}\right)
\end{array}\right) \\
\sum_{i=1}^{n}\left(\alpha_{A}^{2 q}\left(x_{i}\right)+\beta_{A}^{2 q}\left(x_{i}\right)+\delta_{\alpha_{A}}^{2 q}\left(x_{i}\right)+\delta_{\beta_{A}}^{2 q}\left(x_{i}\right)\right) \\
+\sum_{i=1}^{n}\left(\alpha_{B}^{2 q}\left(x_{i}\right)+\beta_{B}^{2 q}\left(x_{i}\right)+\delta_{\alpha_{B}}^{2 q}\left(x_{i}\right)+\delta_{\beta_{B}}^{2 q}\left(x_{i}\right)\right)
\end{array}\right)
\end{aligned}
$$

which satisfy following conditions:

$d_{\mathrm{Cq}-\mathrm{ROF}}^{\mathrm{w} 2}(A, B)=\sum_{i=1}^{n} \omega_{i}\left(\frac{2\left(\begin{array}{c}\alpha_{A}^{q}\left(x_{i}\right) \alpha_{B}^{q}\left(x_{i}\right)+\beta_{A}^{q}\left(x_{i}\right) \beta_{B}^{q}\left(x_{i}\right)+\theta_{A}^{q}\left(x_{i}\right) \theta_{B}^{q}\left(x_{i}\right) \\ +\delta_{\alpha_{A}}^{q}\left(x_{i}\right) \delta_{\alpha_{B}}^{q}\left(x_{i}\right)+\delta_{\beta_{A}}^{q}\left(x_{i}\right) \delta_{\beta_{B}}^{q}\left(x_{i}\right)+\delta_{\theta_{A}}^{q}\left(x_{i}\right) \delta_{\theta_{B}}^{q}\left(x_{i}\right)\end{array}\right)}{\left(\begin{array}{c}\alpha_{A}^{2 q}\left(x_{i}\right)+\beta_{A}^{2 q}\left(x_{i}\right)+\theta_{A}^{2 q}\left(x_{i}\right) \\ +\delta_{\alpha_{A}}^{2 q}\left(x_{i}\right)+\delta_{\beta_{A}}^{2 q}\left(x_{i}\right)+\delta_{\theta_{A}}^{2 q}\left(x_{i}\right)\end{array}\right)+\left(\begin{array}{c}\alpha_{B}^{2 q}\left(x_{i}\right)+\beta_{B}^{2 q}\left(x_{i}\right)+\theta_{B}^{2 q}\left(x_{i}\right) \\ +\delta_{\alpha_{B}}^{2 q}\left(x_{i}\right)+\delta_{\beta_{B}}^{2 q}\left(x_{i}\right)+\delta_{\theta_{B}}^{2 q}\left(x_{i}\right)\end{array}\right)}\right)$

which satisfy following conditions:
4. $0 \leq d_{\mathrm{Cq}-\mathrm{ROF}}^{\mathrm{w} 2}(A, B) \leq 1$
1. $0 \leq d_{\mathrm{Cq}-\mathrm{ROF}}^{3}(A, B) \leq 1$
5. $d_{\mathrm{Cq}-\mathrm{ROF}}^{\mathrm{w} 2}(A, B)=d_{\mathrm{Cq}-\mathrm{ROF}}^{\mathrm{w} 2}(B, A)$
2. $d_{\mathrm{Cq}-\mathrm{ROF}}^{3}(A, B)=d_{\mathrm{Cq}-\mathrm{ROF}}^{3}(B, A)$
1) $d_{\mathrm{Cq}-\mathrm{ROF}}^{\mathrm{W} 2}(A, B)=1$ if $A=B$.
3. $d_{\mathrm{Cq}-\mathrm{ROF}}^{3}(A, B)=1$ if $A=B$. 
Definition 12 A WDSM $d_{\mathrm{Cq}-\mathrm{ROF}}^{\mathrm{w} 3}(A, B)$ for Cq-ROFSs is given by: $d d_{\mathrm{Cq}-\mathrm{ROF}}^{\mathrm{w} 3}(A, B)=1-d_{\mathrm{Cq}-\mathrm{ROF}}^{\mathrm{w} 3}(A, B)$

Definition 13 A DSM $d_{\mathrm{Cq}-\mathrm{ROF}}^{4}(A, B)$ for Cq-ROFSs is given by:

$d_{\mathrm{Cq}-\mathrm{ROF}}^{4}(A, B)=\left(\frac{\sum_{i=1}^{n} 2\left(\begin{array}{c}\alpha_{A}^{q}\left(x_{i}\right) \alpha_{B}^{q}\left(x_{i}\right)+\beta_{A}^{q}\left(x_{i}\right) \beta_{B}^{q}\left(x_{i}\right)+\theta_{A}^{q}\left(x_{i}\right) \theta_{B}^{q}\left(x_{i}\right) \\ +\delta_{\alpha_{A}}^{q}\left(x_{i}\right) \delta_{\alpha_{B}}^{q}\left(x_{i}\right)+\delta_{\beta_{A}}^{q}\left(x_{i}\right) \delta_{\beta_{B}}^{q}\left(x_{i}\right)+\delta_{\theta_{A}}^{q}\left(x_{i}\right) \delta_{\theta_{B}}^{q}\left(x_{i}\right)\end{array}\right)}{\sum_{i=1}^{n}\left(\begin{array}{c}\alpha_{A}^{2 q}\left(x_{i}\right)+\beta_{A}^{2 q}\left(x_{i}\right)+\theta_{A}^{2 q}\left(x_{i}\right) \\ +\delta_{\alpha_{A}}^{2 q}\left(x_{i}\right)+\delta_{\beta_{A}}^{2 q}\left(x_{i}\right)+\delta_{\theta_{A}}^{2 q}\left(x_{i}\right)\end{array}\right)+\sum_{i=1}^{n}\left(\begin{array}{c}\alpha_{B}^{2 q}\left(x_{i}\right)+\beta_{B}^{2 q}\left(x_{i}\right)+\theta_{B}^{2 q}\left(x_{i}\right) \\ +\delta_{\alpha_{B}}^{2 q}\left(x_{i}\right)+\delta_{\beta_{B}}^{2 q}\left(x_{i}\right)+\delta_{\theta_{B}}^{2 q}\left(x_{i}\right)\end{array}\right)}\right)$

$$
\begin{aligned}
& d_{\mathrm{Cq}-\mathrm{ROF}}^{\mathrm{w} 3}(A, B) \\
& =\left(\begin{array}{c}
2 \sum_{i=1}^{n} \omega_{i}^{2 q}\left(\begin{array}{c}
\alpha_{A}^{q}\left(x_{i}\right) \alpha_{B}^{q}\left(x_{i}\right)+\beta_{A}^{q}\left(x_{i}\right) \beta_{B}^{q}\left(x_{i}\right) \\
+\delta_{\alpha_{A}}^{q}\left(x_{i}\right) \delta_{\alpha_{B}}^{q}\left(x_{i}\right)+\delta_{\beta_{A}}^{q}\left(x_{i}\right) \delta_{\beta_{B}}^{q}\left(x_{i}\right)
\end{array}\right) \\
\sum_{i=1}^{n} \omega_{i}^{2 q}\left(\alpha_{A}^{2 q}\left(x_{i}\right)+\beta_{A}^{2 q}\left(x_{i}\right)+\delta_{\alpha_{A}}^{2 q}\left(x_{i}\right)+\delta_{\beta_{A}}^{2 q}\left(x_{i}\right)\right) \\
+\sum_{i=1}^{n} \omega_{i}^{2 q}\left(\alpha_{B}^{2 q}\left(x_{i}\right)+\beta_{B}^{2 q}\left(x_{i}\right)+\delta_{\alpha_{B}}^{2 q}\left(x_{i}\right)+\delta_{\beta_{B}}^{2 q}\left(x_{i}\right)\right)
\end{array}\right)
\end{aligned}
$$

which satisfy following conditions: which satisfy following conditions:

1. $0 \leq d_{\mathrm{Cq}-\mathrm{ROF}}^{4}(A, B) \leq 1$

2. $d_{\mathrm{Cq}-\mathrm{ROF}}^{4}(A, B)=d_{\mathrm{Cq}-\mathrm{ROF}}^{4}(B, A)$

3. $d_{\mathrm{Cq}-\mathrm{ROF}}^{4}(A, B)=1$ if $A=B$.

Definition 14 A WDSM $d_{\mathrm{Cq}-\mathrm{ROF}}^{\mathrm{w} 4}(A, B)$ for Cq-ROFSs is given by:

$d_{\mathrm{Cq}-\mathrm{ROF}}^{\mathrm{w} 4}(A, B)$

$$
=\left(\frac{2 \sum_{i=1}^{n} \omega_{i}^{2 q}\left(\begin{array}{c}
\alpha_{A}^{q}\left(x_{i}\right) \alpha_{B}^{q}\left(x_{i}\right)+\beta_{A}^{q}\left(x_{i}\right) \beta_{B}^{q}\left(x_{i}\right)+\theta_{A}^{q}\left(x_{i}\right) \theta_{B}^{q}\left(x_{i}\right) \\
+\delta_{\alpha_{A}}^{q}\left(x_{i}\right) \delta_{\alpha_{B}}^{q}\left(x_{i}\right)+\delta_{\beta_{A}}^{q}\left(x_{i}\right) \delta_{\beta_{B}}^{q}\left(x_{i}\right)+\delta_{\theta_{A}}^{q}\left(x_{i}\right) \delta_{\theta_{B}}^{q}\left(x_{i}\right)
\end{array}\right)}{\sum_{i=1}^{n} \omega_{i}^{2 q}\left(\begin{array}{c}
\alpha_{A}^{2 q}\left(x_{i}\right)+\beta_{A}^{2 q}\left(x_{i}\right)+\theta_{A}^{2 q}\left(x_{i}\right) \\
+\delta_{\alpha_{A}}^{2 q}\left(x_{i}\right)+\delta_{\beta_{A}}^{2 q}\left(x_{i}\right)+\delta_{\theta_{A}}^{2 q}\left(x_{i}\right)
\end{array}\right)+\sum_{i=1}^{n} \omega_{i}^{2 q}\left(\begin{array}{c}
\alpha_{B}^{2 q}\left(x_{i}\right)+\beta_{B}^{2 q}\left(x_{i}\right)+\theta_{B}^{2 q}\left(x_{i}\right) \\
+\delta_{\alpha_{B}}^{2 q}\left(x_{i}\right)+\delta_{\beta_{B}}^{2 q}\left(x_{i}\right)+\delta_{\theta_{B}}^{2 q}\left(x_{i}\right)
\end{array}\right)}\right)
$$

1. $0 \leq d_{\mathrm{Cq}-\mathrm{ROF}}^{\mathrm{w} 3}(A, B) \leq 1$

2. $d_{\mathrm{Cq}-\mathrm{ROF}}^{\mathrm{w} 3}(A, B)=d_{\mathrm{Cq}-\mathrm{ROF}}^{\mathrm{w} 3}(B, A)$

3. $d_{\mathrm{Cq}-\mathrm{ROF}}^{\mathrm{w} 3}(A, B)=1$ if $A=B$.

Remark 5 If we take $\omega=\left(\frac{1}{n}, \frac{1}{n}, \ldots, \frac{1}{n}\right)^{T}$ then the WDSM for Cq-ROFS reduced to DSM for Cq-ROFS i.e. $d_{\mathrm{Cq}-\mathrm{ROF}}^{\mathrm{w} 3}$ $(A, B)=d_{\mathrm{Cq}-\mathrm{ROF}}^{3}(A, B)$.

Remark 6 The DDM and WDDM for Cq-ROFSs are defined as:

$d d_{\mathrm{Cq}-\mathrm{ROF}}^{3}(A, B)=1-d_{\mathrm{Cq}-\mathrm{ROF}}^{3}(A, B)$ which satisfy following conditions:

1. $0 \leq d_{\mathrm{Cq}-\mathrm{ROF}}^{\mathrm{w} 4}(A, B) \leq 1$

2. $d_{\mathrm{Cq}-\mathrm{ROF}}^{\mathrm{w} 4}(A, B)=d_{\mathrm{Cq}-\mathrm{ROF}}^{\mathrm{w} 4}(B, A)$

3. $d_{\mathrm{Cq}-\mathrm{ROF}}^{\mathrm{w} 4}(A, B)=1$ if $A=B$.

Remark 7 If we take $\omega=\left(\frac{1}{n}, \frac{1}{n}, \ldots, \frac{1}{n}\right)^{T}$ then the WDSM for Cq-ROFS reduced to DSM for Cq-ROFS i.e. $d_{\mathrm{Cq}-\mathrm{ROF}}^{\mathrm{w} 4}$ $(A, B)=d_{\mathrm{Cq}-\mathrm{ROF}}^{4}(A, B)$.

Remark 8 The DDM and WDDM for Cq-ROFSs are defined as: 
$d d_{\mathrm{Cq}-\mathrm{ROF}}^{4}(A, B)=1-d_{\mathrm{Cq}-\mathrm{ROF}}^{4}(A, B)$

$d d_{\mathrm{Cq}-\mathrm{ROF}}^{\mathrm{w} 4}(A, B)=1-d_{\mathrm{Cq}-\mathrm{ROF}}^{\mathrm{w} 4}(A, B)$

\section{Generalized dice similarity measures for Cq-ROFSs}

In this sub-section, we propose the notion of generalized DSM (GDSM) and weighted GDSM (WGDSM) for CqROFSs. The special cases of the proposed approaches are also discussed in detail. Let $Y \in[0,1]$ be a real parameter.

Definition 15 For two Cq-ROFSs, a GDSM $d_{\mathrm{Cq}-\mathrm{ROF}}^{\mathrm{G} 1}(A, B)$ is given by:

$$
\begin{aligned}
& d_{\mathrm{Cq}-\mathrm{ROF}}^{\mathrm{G} 1}(A, B) \\
& =\frac{1}{n} \sum_{i=1}^{n}\left(\begin{array}{c}
\left(\begin{array}{c}
\alpha_{A}^{q}\left(x_{i}\right) \alpha_{B}^{q}\left(x_{i}\right)+\beta_{A}^{q}\left(x_{i}\right) \beta_{B}^{q}\left(x_{i}\right) \\
+\delta_{\alpha_{A}}^{q}\left(x_{i}\right) \delta_{\alpha_{B}}^{q}\left(x_{i}\right)+\delta_{\beta_{A}}^{q}\left(x_{i}\right) \delta_{\beta_{B}}^{q}\left(x_{i}\right)
\end{array}\right) \\
\Upsilon\left(\alpha_{A}^{2 q}\left(x_{i}\right)+\beta_{A}^{2 q}\left(x_{i}\right)+\delta_{\alpha_{A}}^{2 q}\left(x_{i}\right)+\delta_{\beta_{A}}^{2 q}\left(x_{i}\right)\right) \\
+(1-\Upsilon)\left(\alpha_{B}^{2 q}\left(x_{i}\right)+\beta_{B}^{2 q}\left(x_{i}\right)+\delta_{\alpha_{B}}^{2 q}\left(x_{i}\right)+\delta_{\beta_{B}}^{2 q}\left(x_{i}\right)\right)
\end{array}\right)
\end{aligned}
$$

where $Y \in[0,1]$ is the parameter. Equation (22) satisfies the following conditions:

1. $0 \leq d_{\mathrm{Cq}-\mathrm{ROF}}^{\mathrm{G} 1}(A, B) \leq 1$

2. $d_{\mathrm{Cq}-\mathrm{ROF}}^{\mathrm{G} 1}(A, B)=d_{\mathrm{Cq}-\mathrm{ROF}}^{\mathrm{G} 1}(B, A)$

3. $d_{\mathrm{Cq}-\mathrm{ROF}}^{\mathrm{G} 1}(A, B)=1$ if $A=B$.

If we consider the value of $\Upsilon=0$, then

$$
\begin{aligned}
& d_{\mathrm{Cq}-\mathrm{ROF}}^{\mathrm{G} 1}(A, B) \\
& =\frac{1}{n} \sum_{i=1}^{n}\left(\frac{\left(\begin{array}{c}
\alpha_{A}^{q}\left(x_{i}\right) \alpha_{B}^{q}\left(x_{i}\right)+\beta_{A}^{q}\left(x_{i}\right) \beta_{B}^{q}\left(x_{i}\right) \\
+\delta_{\alpha_{A}}^{q}\left(x_{i}\right) \delta_{\alpha_{B}}^{q}\left(x_{i}\right)+\delta_{\beta_{A}}^{q}\left(x_{i}\right) \delta_{\beta_{B}}^{q}\left(x_{i}\right)
\end{array}\right)}{\left(\alpha_{B}^{2 q}\left(x_{i}\right)+\beta_{B}^{2 q}\left(x_{i}\right)+\delta_{\alpha_{B}}^{2 q}\left(x_{i}\right)+\delta_{\beta_{B}}^{2 q}\left(x_{i}\right)\right)}\right)
\end{aligned}
$$

On the other hand, if we consider $\Upsilon=1$, then
$d_{\mathrm{Cq}-\mathrm{ROF}}^{G 1}(A, B)$

$$
=\frac{1}{n} \sum_{i=1}^{n}\left(\frac{\left(\begin{array}{c}
\alpha_{A}^{q}\left(x_{i}\right) \alpha_{B}^{q}\left(x_{i}\right)+\beta_{A}^{q}\left(x_{i}\right) \beta_{B}^{q}\left(x_{i}\right) \\
+\delta_{\alpha_{A}}^{q}\left(x_{i}\right) \delta_{\alpha_{B}}^{q}\left(x_{i}\right)+\delta_{\beta_{A}}^{q}\left(x_{i}\right) \delta_{\beta_{B}}^{q}\left(x_{i}\right)
\end{array}\right)}{\left(\alpha_{A}^{2 q}\left(x_{i}\right)+\beta_{A}^{2 q}\left(x_{i}\right)+\delta_{\alpha_{A}}^{2 q}\left(x_{i}\right)+\delta_{\beta_{A}}^{2 q}\left(x_{i}\right)\right)}\right)
$$

is called asymmetric similarity measures or projection similarity measures.

Definition 16 A WGDSM $d_{\mathrm{Cq}-\mathrm{ROF}}^{\mathrm{wG}}(A, B)$ for Cq-ROFSs is given by:

$d_{\mathrm{Cq}-\mathrm{ROF}}^{\mathrm{wG} 1}(A, B)$

$$
=\sum_{i=1}^{n} \omega_{i}\left(\begin{array}{c}
\left(\begin{array}{c}
\alpha_{A}^{q}\left(x_{i}\right) \alpha_{B}^{q}\left(x_{i}\right)+\beta_{A}^{q}\left(x_{i}\right) \beta_{B}^{q}\left(x_{i}\right) \\
+\delta_{\alpha_{A}}^{q}\left(x_{i}\right) \delta_{\alpha_{B}}^{q}\left(x_{i}\right)+\delta_{\beta_{A}}^{q}\left(x_{i}\right) \delta_{\beta_{B}}^{q}\left(x_{i}\right)
\end{array}\right) \\
\Upsilon\left(\alpha_{A}^{2 q}\left(x_{i}\right)+\beta_{A}^{2 q}\left(x_{i}\right)+\delta_{\alpha_{A}}^{2 q}\left(x_{i}\right)+\delta_{\beta_{A}}^{2 q}\left(x_{i}\right)\right) \\
+(1-\Upsilon)\left(\alpha_{B}^{2 q}\left(x_{i}\right)+\beta_{B}^{2 q}\left(x_{i}\right)+\delta_{\alpha_{B}}^{2 q}\left(x_{i}\right)+\delta_{\beta_{B}}^{2 q}\left(x_{i}\right)\right)
\end{array}\right)
$$

where $0 \leq \Upsilon \leq 1$. Further, $d_{\mathrm{Cq}-\mathrm{ROF}}^{\mathrm{wG} 1}$ satisfy following conditions:

1. $0 \leq d_{\mathrm{Cq}-\mathrm{ROF}}^{\mathrm{wG} 1}(A, B) \leq 1$

2. $d_{\mathrm{Cq}-\mathrm{ROF}}^{\mathrm{wG} 1}(A, B)=d_{\mathrm{Cq}-\mathrm{ROF}}^{\mathrm{wG} 1}(B, A)$

3. $d_{\mathrm{Cq}-\mathrm{ROF}}^{\mathrm{wG1}}(A, B)=1$ if $A=B$.

If we set the value of $\Upsilon=0$, then $d_{\mathrm{Cq}-\mathrm{ROF}}^{\mathrm{wG} 1}(A, B)$

$$
=\sum_{i=1}^{n} \omega_{i}\left(\frac{\left(\begin{array}{c}
\alpha_{A}^{q}\left(x_{i}\right) \alpha_{B}^{q}\left(x_{i}\right)+\beta_{A}^{q}\left(x_{i}\right) \beta_{B}^{q}\left(x_{i}\right) \\
+\delta_{\alpha_{A}}^{q}\left(x_{i}\right) \delta_{\alpha_{B}}^{q}\left(x_{i}\right)+\delta_{\beta_{A}}^{q}\left(x_{i}\right) \delta_{\beta_{B}}^{q}\left(x_{i}\right)
\end{array}\right)}{\left(\alpha_{B}^{2 q}\left(x_{i}\right)+\beta_{B}^{2 q}\left(x_{i}\right)+\delta_{\alpha_{B}}^{2 q}\left(x_{i}\right)+\delta_{\beta_{B}}^{2 q}\left(x_{i}\right)\right)}\right)
$$

while when $\Upsilon=1$, then

$d_{\mathrm{Cq}-\mathrm{ROF}}^{\mathrm{wG} 1}(A, B)$

$$
=\sum_{i=1}^{n} \omega_{i}\left(\frac{\left(\begin{array}{c}
\alpha_{A}^{q}\left(x_{i}\right) \alpha_{B}^{q}\left(x_{i}\right)+\beta_{A}^{q}\left(x_{i}\right) \beta_{B}^{q}\left(x_{i}\right) \\
+\delta_{\alpha_{A}}^{q}\left(x_{i}\right) \delta_{\alpha_{B}}^{q}\left(x_{i}\right)+\delta_{\beta_{A}}^{q}\left(x_{i}\right) \delta_{\beta_{B}}^{q}\left(x_{i}\right)
\end{array}\right)}{\left(\alpha_{A}^{2 q}\left(x_{i}\right)+\beta_{A}^{2 q}\left(x_{i}\right)+\delta_{\alpha_{A}}^{2 q}\left(x_{i}\right)+\delta_{\beta_{A}}^{2 q}\left(x_{i}\right)\right)}\right)
$$

is called asymmetric similarity measures or projection similarity measures. 
Remark 9 By changing $\Upsilon=0.5$, the Eqs. (22) and (25) are reduced into Eqs. (6) and (7).

Remark 10 If we take $\omega=\left(\frac{1}{n}, \frac{1}{n}, \ldots, \frac{1}{n}\right)^{T}$ then the WGDSM for Cq-ROFS reduced to GDSM for Cq-ROFS i.e. $d_{\mathrm{Cq}-\mathrm{ROF}}^{\mathrm{wG}}(A, B)=d_{\mathrm{Cq}-\mathrm{ROF}}^{\mathrm{G} 1}(A, B)$.

Remark 11 The generalized dice distance measure (GDDM) and weighted generalized dice distance measure (WGDDM) for Cq-ROFSs are defined as:

$d d_{\mathrm{Cq}-\mathrm{ROF}}^{\mathrm{G} 1}(A, B)=1-d_{\mathrm{Cq}-\mathrm{ROF}}^{\mathrm{G} 1}(A, B)$

$d d_{\mathrm{Cq}-\mathrm{ROF}}^{\mathrm{wG} 1}(A, B)=1-d_{\mathrm{Cq}-\mathrm{ROF}}^{\mathrm{wG} 1}(A, B)$

Definition 17 A GDSM $d_{\mathrm{Cq}-\mathrm{ROF}}^{\mathrm{G} 2}(A, B)$ for Cq-ROFSs is given by:
$d_{C q-R O F}^{G 2}(A, B)$

$$
=\frac{1}{n} \sum_{i=1}^{n}\left(\frac{\left(\begin{array}{c}
\alpha_{A}^{q}\left(x_{i}\right) \alpha_{B}^{q}\left(x_{i}\right)+\beta_{A}^{q}\left(x_{i}\right) \beta_{B}^{q}\left(x_{i}\right)+\theta_{A}^{q}\left(x_{i}\right) \theta_{B}^{q}\left(x_{i}\right)+ \\
\delta_{\alpha_{A}}^{q}\left(x_{i}\right) \delta_{\alpha_{B}}^{q}\left(x_{i}\right)+\delta_{\beta_{A}}^{q}\left(x_{i}\right) \delta_{\beta_{B}}^{q}\left(x_{i}\right)+\delta_{\theta_{A}}^{q}\left(x_{i}\right) \delta_{\theta_{B}}^{q}\left(x_{i}\right)
\end{array}\right)}{\left(\begin{array}{l}
\alpha_{B}^{2 q}\left(x_{i}\right)+\beta_{B}^{2 q}\left(x_{i}\right)+\theta_{B}^{2 q}\left(x_{i}\right)+ \\
\delta_{\alpha_{B}}^{2 q}\left(x_{i}\right)+\delta_{\beta_{B}}^{2 q}\left(x_{i}\right)+\delta_{\theta_{B}}^{2 q}\left(x_{i}\right)
\end{array}\right)}\right)
$$

If we consider the value of $\Upsilon=1$, then $d_{\mathrm{Cq}-\mathrm{ROF}}^{\mathrm{G} 2}(A, B)$

$$
=\frac{1}{n} \sum_{i=1}^{n}\left(\frac{\left(\begin{array}{c}
\alpha_{A}^{q}\left(x_{i}\right) \alpha_{B}^{q}\left(x_{i}\right)+\beta_{A}^{q}\left(x_{i}\right) \beta_{B}^{q}\left(x_{i}\right)+\theta_{A}^{q}\left(x_{i}\right) \theta_{B}^{q}\left(x_{i}\right)+ \\
\delta_{\alpha_{A}}^{q}\left(x_{i}\right) \delta_{\alpha_{B}}^{q}\left(x_{i}\right)+\delta_{\beta_{A}}^{q}\left(x_{i}\right) \delta_{\beta_{B}}^{q}\left(x_{i}\right)+\delta_{\theta_{A}}^{q}\left(x_{i}\right) \delta_{\theta_{B}}^{q}\left(x_{i}\right)
\end{array}\right)}{\left(\begin{array}{c}
\alpha_{A}^{2 q}\left(x_{i}\right)+\beta_{A}^{2 q}\left(x_{i}\right)+\theta_{A}^{2 q}\left(x_{i}\right) \\
+\delta_{\alpha_{A}}^{2 q}\left(x_{i}\right)+\delta_{\beta_{A}}^{2 q}\left(x_{i}\right)+\delta_{\theta_{A}}^{2 q}\left(x_{i}\right)
\end{array}\right)}\right)
$$

is called asymmetric similarity measures or projection similarity measures.

$d_{\mathrm{Cq}-\mathrm{ROF}}^{\mathrm{G} 2}(A, B)$

$$
=\frac{1}{n} \sum_{i=1}^{n}\left(\frac{\alpha_{A}^{q}\left(x_{i}\right) \alpha_{B}^{q}\left(x_{i}\right)+\beta_{A}^{q}\left(x_{i}\right) \beta_{B}^{q}\left(x_{i}\right)+\theta_{A}^{q}\left(x_{i}\right) \theta_{B}^{q}\left(x_{i}\right)+}{\delta_{\alpha_{A}}^{q}\left(x_{i}\right) \delta_{\alpha_{B}}^{q}\left(x_{i}\right)+\delta_{\beta_{A}}^{q}\left(x_{i}\right) \delta_{\beta_{B}}^{q}\left(x_{i}\right)+\delta_{\theta_{A}}^{q}\left(x_{i}\right) \delta_{\theta_{B}}^{q}\left(x_{i}\right)}\left(\begin{array}{c}
2 q \\
\Upsilon\left(\begin{array}{c}
\alpha_{A}^{2 q}\left(x_{i}\right)+\beta_{A}^{2 q}\left(x_{i}\right)+\theta_{A}^{2 q}\left(x_{i}\right)+ \\
\delta_{\alpha_{A}}^{2 q}\left(x_{i}\right)+\delta_{\beta_{A}}^{2 q}\left(x_{i}\right)+\delta_{\theta_{A}}^{2 q}\left(x_{i}\right)
\end{array}\right)+(1-\Upsilon)\left(\begin{array}{c}
\alpha_{B}^{2 q}\left(x_{i}\right)+\beta_{B}^{2 q}\left(x_{i}\right)+\theta_{B}^{2 q}\left(x_{i}\right)+ \\
\delta_{\alpha_{B}}^{2 q}\left(x_{i}\right)+\delta_{\beta_{B}}^{2 q}\left(x_{i}\right)+\delta_{\theta_{B}}^{2 q}\left(x_{i}\right)
\end{array}\right)
\end{array}\right)\right.
$$

which satisfy following conditions:

Definition 18 A WGDSM $d_{\mathrm{Cq}-\mathrm{ROF}}^{\mathrm{wG} 2}(A, B)$ for Cq-ROFSs is given by:

$d_{\mathrm{Cq}-\mathrm{ROF}}^{\mathrm{wG} 2}(A, B)$

$$
=\sum_{i=1}^{n} \omega_{i}\left(\frac{\left.\begin{array}{c}
\alpha_{A}^{q}\left(x_{i}\right) \alpha_{B}^{q}\left(x_{i}\right)+\beta_{A}^{q}\left(x_{i}\right) \beta_{B}^{q}\left(x_{i}\right)+\theta_{A}^{q}\left(x_{i}\right) \theta_{B}^{q}\left(x_{i}\right) \\
+\delta_{\alpha_{A}}^{q}\left(x_{i}\right) \delta_{\alpha_{B}}^{q}\left(x_{i}\right)+\delta_{\beta_{A}}^{q}\left(x_{i}\right) \delta_{\beta_{B}}^{q}\left(x_{i}\right)+\delta_{\theta_{A}}^{q}\left(x_{i}\right) \delta_{\theta_{B}}^{q}\left(x_{i}\right)
\end{array}\right)}{\Upsilon\left(\begin{array}{c}
\alpha_{A}^{2 q}\left(x_{i}\right)+\beta_{A}^{2 q}\left(x_{i}\right)+\theta_{A}^{2 q}\left(x_{i}\right) \\
+\delta_{\alpha_{A}}^{2 q}\left(x_{i}\right)+\delta_{\beta_{A}}^{2 q}\left(x_{i}\right)+\delta_{\theta_{A}}^{2 q}\left(x_{i}\right)
\end{array}\right)+(1-\Upsilon)\left(\begin{array}{c}
\alpha_{B}^{2 q}\left(x_{i}\right)+\beta_{B}^{2 q}\left(x_{i}\right)+\theta_{B}^{2 q}\left(x_{i}\right) \\
+\delta_{\alpha_{B}}^{2 q}\left(x_{i}\right)+\delta_{\beta_{B}}^{2 q}\left(x_{i}\right)+\delta_{\theta_{B}}^{2 q}\left(x_{i}\right)
\end{array}\right)}\right)
$$

1.

$$
0 \leq d_{C q-R O F}^{G 2}(A, B) \leq 1
$$

2. $d_{C q-R O F}^{G 2}(A, B)=d_{C q-R O F}^{G 2}(B, A)$

3. $d_{C q-R O F}^{G 2}(A, B)=\operatorname{lif} A=B$.

If we consider the value of $\Upsilon=0$, then where $0 \leq \Upsilon \leq 1$. Further, $d_{\mathrm{Cq}-\mathrm{ROF}}^{\mathrm{G} 2}(A, B)$ satisfy following conditions:

1. $0 \leq d_{\mathrm{Cq}-\operatorname{ROF}}^{\mathrm{WG} 2}(A, B) \leq 1$

2. $d_{\mathrm{Cq}-\mathrm{ROF}}^{\mathrm{wG} 2}(A, B)=d_{\mathrm{Cq}-\mathrm{ROF}}^{\mathrm{wG}}(B, A)$

3. $d_{\mathrm{Cq}-\mathrm{ROF}}^{\mathrm{wG} 2}(A, B)=1$ if $A=B$. 
If we consider the value of $\Upsilon=0$, then

$$
\begin{aligned}
& d_{\mathrm{Cq}-\mathrm{ROF}}^{\mathrm{wG}}(A, B) \\
& =\sum_{i=1}^{n} \omega_{i}\left(\frac{\left(\begin{array}{c}
\alpha_{A}^{q}\left(x_{i}\right) \alpha_{B}^{q}\left(x_{i}\right)+\beta_{A}^{q}\left(x_{i}\right) \beta_{B}^{q}\left(x_{i}\right)+\theta_{A}^{q}\left(x_{i}\right) \theta_{B}^{q}\left(x_{i}\right) \\
+\delta_{\alpha_{A}}^{q}\left(x_{i}\right) \delta_{\alpha_{B}}^{q}\left(x_{i}\right)+\delta_{\beta_{A}}^{q}\left(x_{i}\right) \delta_{\beta_{B}}^{q}\left(x_{i}\right)+\delta_{\theta_{A}}^{q}\left(x_{i}\right) \delta_{\theta_{B}}^{q}\left(x_{i}\right)
\end{array}\right)}{\left(\begin{array}{c}
\alpha_{B}^{2 q}\left(x_{i}\right)+\beta_{B}^{2 q}\left(x_{i}\right)+\theta_{B}^{2 q}\left(x_{i}\right) \\
+\delta_{\alpha_{B}}^{2 q}\left(x_{i}\right)+\delta_{\beta_{B}}^{2 q}\left(x_{i}\right)+\delta_{\theta_{B}}^{2 q}\left(x_{i}\right)
\end{array}\right)}\right)
\end{aligned}
$$

If we consider the value of $\Upsilon=1$, then

$$
\begin{aligned}
& d_{\mathrm{Cq}-\mathrm{ROF}}^{\mathrm{wG} 2}(A, B) \\
& =\sum_{i=1}^{n} \omega_{i}\left(\frac{\left(\begin{array}{c}
\alpha_{A}^{q}\left(x_{i}\right) \alpha_{B}^{q}\left(x_{i}\right)+\beta_{A}^{q}\left(x_{i}\right) \beta_{B}^{q}\left(x_{i}\right)+\theta_{A}^{q}\left(x_{i}\right) \theta_{B}^{q}\left(x_{i}\right) \\
+\delta_{\alpha_{A}}^{q}\left(x_{i}\right) \delta_{\alpha_{B}}^{q}\left(x_{i}\right)+\delta_{\beta_{A}}^{q}\left(x_{i}\right) \delta_{\beta_{B}}^{q}\left(x_{i}\right)+\delta_{\theta_{A}}^{q}\left(x_{i}\right) \delta_{\theta_{B}}^{q}\left(x_{i}\right)
\end{array}\right)}{\left(\begin{array}{c}
\alpha_{A}^{2 q}\left(x_{i}\right)+\beta_{A}^{2 q}\left(x_{i}\right)+\theta_{A}^{2 q}\left(x_{i}\right) \\
+\delta_{\alpha_{A}}^{2 q}\left(x_{i}\right)+\delta_{\beta_{A}}^{2 q}\left(x_{i}\right)+\delta_{\theta_{A}}^{2 q}\left(x_{i}\right)
\end{array}\right)}\right)
\end{aligned}
$$

is called asymmetric similarity measures or projection similarity measures.

Remark 12 By changing $\Upsilon=0.5$, the Eqs. (30) and (33) are reduced into Eqs. (10) and (11).

Remark 13 If we take $\omega=\left(\frac{1}{n}, \frac{1}{n}, \ldots, \frac{1}{n}\right)^{T}$ then the WGDSM for Cq-ROFS reduced to GDSM for Cq-ROFS i.e. $\mathrm{d}_{\mathrm{Cq}-\mathrm{ROF}}^{\mathrm{wG}}(A, B)=d_{\mathrm{Cq}-\mathrm{ROF}}^{\mathrm{G} 2}(A, B)$.

Remark 14 The GDDM and WGDDM for Cq-ROFSs are defined as:

$d d_{\mathrm{Cq}-\mathrm{ROF}}^{\mathrm{G} 2}(A, B)=1-d_{\mathrm{Cq}-\mathrm{ROF}}^{\mathrm{G} 2}(A, B)$

$d d_{\mathrm{Cq}-\mathrm{ROF}}^{\mathrm{wG} 2}(A, B)=1-d_{\mathrm{Cq}-\mathrm{ROF}}^{\mathrm{wG} 2}(A, B)$

Definition 19 A GDSM $d_{\mathrm{Cq}-\mathrm{ROF}}^{\mathrm{G} 3}(A, B)$ for Cq-ROFSs is gavemoby! $B$ )

$$
=\left(\begin{array}{c}
\sum_{i=1}^{n}\left(\begin{array}{c}
\alpha_{A}^{q}\left(x_{i}\right) \alpha_{B}^{q}\left(x_{i}\right)+\beta_{A}^{q}\left(x_{i}\right) \beta_{B}^{q}\left(x_{i}\right) \\
+\delta_{\alpha_{A}}^{q}\left(x_{i}\right) \delta_{\alpha_{B}}^{q}\left(x_{i}\right)+\delta_{\beta_{A}}^{q}\left(x_{i}\right) \delta_{\beta_{B}}^{q}\left(x_{i}\right)
\end{array}\right) \\
\Upsilon \sum_{i=1}^{n}\left(\alpha_{A}^{2 q}\left(x_{i}\right)+\beta_{A}^{2 q}\left(x_{i}\right)+\delta_{\alpha_{A}}^{2 q}\left(x_{i}\right)+\delta_{\beta_{A}}^{2 q}\left(x_{i}\right)\right) \\
+(1-\Upsilon) \sum_{i=1}^{n}\left(\alpha_{B}^{2 q}\left(x_{i}\right)+\beta_{B}^{2 q}\left(x_{i}\right)+\delta_{\alpha_{B}}^{2 q}\left(x_{i}\right)+\delta_{\beta_{B}}^{2 q}\left(x_{i}\right)\right)
\end{array}\right)
$$

which satisfy following conditions:

1. $0 \leq d_{\mathrm{Cq}-\mathrm{ROF}}^{\mathrm{G} 3}(A, B) \leq 1$

2. $d_{\mathrm{Cq}-\mathrm{ROF}}^{\mathrm{G} 3}(A, B)=d_{\mathrm{Cq}-\mathrm{ROF}}^{\mathrm{G} 3}(B, A)$

3. $d_{\mathrm{Cq}-\mathrm{ROF}}^{\mathrm{G} 3}(A, B)=1$ if $A=B$.
If we consider the value of $\Upsilon=0$, then

$$
\begin{aligned}
& d_{\mathrm{Cq}-\mathrm{ROF}}^{\mathrm{G} 3}(A, B) \\
& =\left(\frac{\sum_{i=1}^{n}\left(\begin{array}{c}
\alpha_{A}^{q}\left(x_{i}\right) \alpha_{B}^{q}\left(x_{i}\right)+\beta_{A}^{q}\left(x_{i}\right) \beta_{B}^{q}\left(x_{i}\right) \\
+\delta_{\alpha_{A}}^{q}\left(x_{i}\right) \delta_{\alpha_{B}}^{q}\left(x_{i}\right)+\delta_{\beta_{A}}^{q}\left(x_{i}\right) \delta_{\beta_{B}}^{q}\left(x_{i}\right)
\end{array}\right)}{\sum_{i=1}^{n}\left(\alpha_{B}^{2 q}\left(x_{i}\right)+\beta_{B}^{2 q}\left(x_{i}\right)+\delta_{\alpha_{B}}^{2 q}\left(x_{i}\right)+\delta_{\beta_{B}}^{2 q}\left(x_{i}\right)\right)}\right)
\end{aligned}
$$

If we consider the value of $\Upsilon=1$, then

$$
\begin{aligned}
& d_{\mathrm{Cq}-\mathrm{ROF}}^{\mathrm{G} 3}(A, B) \\
& =\left(\frac{\sum_{i=1}^{n}\left(\begin{array}{c}
\alpha_{A}^{q}\left(x_{i}\right) \alpha_{B}^{q}\left(x_{i}\right)+\beta_{A}^{q}\left(x_{i}\right) \beta_{B}^{q}\left(x_{i}\right) \\
+\delta_{\alpha_{A}}^{q}\left(x_{i}\right) \delta_{\alpha_{B}}^{q}\left(x_{i}\right)+\delta_{\beta_{A}}^{q}\left(x_{i}\right) \delta_{\beta_{B}}^{q}\left(x_{i}\right)
\end{array}\right)}{\sum_{i=1}^{n}\left(\alpha_{A}^{2 q}\left(x_{i}\right)+\beta_{A}^{2 q}\left(x_{i}\right)+\delta_{\alpha_{A}}^{2 q}\left(x_{i}\right)+\delta_{\beta_{A}}^{2 q}\left(x_{i}\right)\right)}\right)
\end{aligned}
$$

is called asymmetric similarity measures or projection similarity measures.

Definition 20 A WGDSM $d_{\mathrm{Cq}-\mathrm{ROF}}^{\mathrm{wG} 3}(A, B)$ for Cq-ROFSs is given by:

$$
\begin{aligned}
& d_{\mathrm{Cq}-\mathrm{ROF}}^{\mathrm{wG} 3}(A, B) \\
& =\left(\begin{array}{c}
\frac{\sum_{i=1}^{n} \omega_{i}^{2 q}\left(\begin{array}{c}
\alpha_{A}^{q}\left(x_{i}\right) \alpha_{B}^{q}\left(x_{i}\right)+\beta_{A}^{q}\left(x_{i}\right) \beta_{B}^{q}\left(x_{i}\right) \\
+\delta_{\alpha_{A}}^{q}\left(x_{i}\right) \delta_{\alpha_{B}}^{q}\left(x_{i}\right)+\delta_{\beta_{A}}^{q}\left(x_{i}\right) \delta_{\beta_{B}}^{q}\left(x_{i}\right)
\end{array}\right)}{\Upsilon \sum_{i=1}^{n} \omega_{i}^{2 q}\left(\alpha_{A}^{2 q}\left(x_{i}\right)+\beta_{A}^{2 q}\left(x_{i}\right)+\delta_{\alpha_{A}}^{2 q}\left(x_{i}\right)+\delta_{\beta_{A}}^{2 q}\left(x_{i}\right)\right)} \\
+(1-\Upsilon) \sum_{i=1}^{n} \omega_{i}^{2 q}\left(\alpha_{B}^{2 q}\left(x_{i}\right)+\beta_{B}^{2 q}\left(x_{i}\right)+\delta_{\alpha_{B}}^{2 q}\left(x_{i}\right)+\delta_{\beta_{B}}^{2 q}\left(x_{i}\right)\right)
\end{array}\right)
\end{aligned}
$$

which satisfy following conditions:

1. $0 \leq d_{\mathrm{Cq}-\operatorname{ROF}}^{\mathrm{wG} 3}(A, B) \leq 1$

2. $\quad d_{\mathrm{Cq}-\mathrm{ROF}}^{\mathrm{wG}}(A, B)=d_{\mathrm{Cq}-\mathrm{ROF}}^{\mathrm{wG} 3}(B, A)$

3. $d_{\mathrm{Cq}-\mathrm{ROF}}^{\mathrm{wG} 3}(A, B)=1$ if $A=B$.

If we consider the value of $\Upsilon=0$, then 
$d_{\mathrm{Cq}-\mathrm{ROF}}^{\mathrm{wG} 3}(A, B)$

$$
=\left(\frac{\sum_{i=1}^{n} \omega_{i}^{2 q}\left(\begin{array}{c}
\alpha_{A}^{q}\left(x_{i}\right) \alpha_{B}^{q}\left(x_{i}\right)+\beta_{A}^{q}\left(x_{i}\right) \beta_{B}^{q}\left(x_{i}\right) \\
+\delta_{\alpha_{A}}^{q}\left(x_{i}\right) \delta_{\alpha_{B}}^{q}\left(x_{i}\right)+\delta_{\beta_{A}}^{q}\left(x_{i}\right) \delta_{\beta_{B}}^{q}\left(x_{i}\right)
\end{array}\right)}{\sum_{i=1}^{n} \omega_{i}^{2 q}\left(\alpha_{B}^{2 q}\left(x_{i}\right)+\beta_{B}^{2 q}\left(x_{i}\right)+\delta_{\alpha_{B}}^{2 q}\left(x_{i}\right)+\delta_{\beta_{B}}^{2 q}\left(x_{i}\right)\right)}\right)
$$

If we consider the value of $\Upsilon=1$, then $d_{\mathrm{Cq}-\mathrm{ROF}}^{\mathrm{wG} 3}(A, B)$

$$
=\left(\frac{\sum_{i=1}^{n} \omega_{i}^{2 q}\left(\begin{array}{c}
\alpha_{A}^{q}\left(x_{i}\right) \alpha_{B}^{q}\left(x_{i}\right)+\beta_{A}^{q}\left(x_{i}\right) \beta_{B}^{q}\left(x_{i}\right) \\
+\delta_{\alpha_{A}}^{q}\left(x_{i}\right) \delta_{\alpha_{B}}^{q}\left(x_{i}\right)+\delta_{\beta_{A}}^{q}\left(x_{i}\right) \delta_{\beta_{B}}^{q}\left(x_{i}\right)
\end{array}\right)}{\sum_{i=1}^{n} \omega_{i}^{2 q}\left(\alpha_{A}^{2 q}\left(x_{i}\right)+\beta_{A}^{2 q}\left(x_{i}\right)+\delta_{\alpha_{A}}^{2 q}\left(x_{i}\right)+\delta_{\beta_{A}}^{2 q}\left(x_{i}\right)\right)}\right)
$$

is called asymmetric similarity measures or projection similarity measures.

Remark 15 By changing $\Upsilon=0.5$, the Eqs. (38) and (41) are reduced into Eqs. (14) and (15).

Remark 16 If we take $\omega=\left(\frac{1}{n}, \frac{1}{n}, \ldots, \frac{1}{n}\right)^{T}$ then the WGDSM for Cq-ROFS reduced to GDSM for Cq-ROFS i.e. $d_{\mathrm{Cq}-\mathrm{ROF}}^{\mathrm{wG}}(A, B)=d_{\mathrm{Cq}-\mathrm{ROF}}^{\mathrm{G} 3}(A, B)$.

Remark 17 The GDDM and WGDDM for Cq-ROFSs are deformed as: $(A, B)=1-d_{\mathrm{Cq}-\mathrm{ROF}}^{\mathrm{G} 3}(A, B)$

$d d_{\mathrm{Cq}-\mathrm{ROF}}^{\mathrm{wG} 3}(A, B)=1-d_{\mathrm{Cq}-\mathrm{ROF}}^{\mathrm{wG} 3}(A, B)$

Definition 21 A GDSM $d_{\mathrm{Cq}-\mathrm{ROF}}^{\mathrm{G} 4}(A, B)$ for Cq-ROFSs is

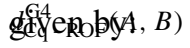

$$
=\left(\begin{array}{c}
\sum_{i=1}^{n}\left(\begin{array}{c}
\alpha_{A}^{q}\left(x_{i}\right) \alpha_{B}^{q}\left(x_{i}\right)+\beta_{A}^{q}\left(x_{i}\right) \beta_{B}^{q}\left(x_{i}\right)+\theta_{A}^{q}\left(x_{i}\right) \theta_{B}^{q}\left(x_{i}\right)+ \\
\delta_{\alpha_{A}}^{q}\left(x_{i}\right) \delta_{\alpha_{B}}^{q}\left(x_{i}\right)+\delta_{\beta_{A}}^{q}\left(x_{i}\right) \delta_{\beta_{B}}^{q}\left(x_{i}\right)+\delta_{\theta_{A}}^{q}\left(x_{i}\right) \delta_{\theta_{B}}^{q}\left(x_{i}\right)
\end{array}\right) \\
\Upsilon \sum_{i=1}^{n}\left(\begin{array}{l}
\alpha_{A}^{2 q}\left(x_{i}\right)+\beta_{A}^{2 q}\left(x_{i}\right)+\theta_{A}^{2 q}\left(x_{i}\right)+ \\
\delta_{\alpha_{A}}^{2 q}\left(x_{i}\right)+\delta_{\beta_{A}}^{2 q}\left(x_{i}\right)+\delta_{\theta_{A}}^{2 q}\left(x_{i}\right)
\end{array}\right) \\
+(1-\Upsilon) \sum_{i=1}^{n}\left(\begin{array}{l}
\alpha_{B}^{2 q}\left(x_{i}\right)+\beta_{B}^{2 q}\left(x_{i}\right)+\theta_{B}^{2 q}\left(x_{i}\right)+ \\
\delta_{\alpha_{B}}^{2 q}\left(x_{i}\right)+\delta_{\beta_{B}}^{2 q}\left(x_{i}\right)+\delta_{\theta_{B}}^{2 q}\left(x_{i}\right)
\end{array}\right)
\end{array}\right)
$$

which satisfy following conditions:

1. $0 \leq d_{\mathrm{Cq}-\mathrm{ROF}}^{\mathrm{G} 4}(A, B) \leq 1$

2. $d_{\mathrm{Cq}-\mathrm{ROF}}^{\mathrm{G} 4}(A, B)=d_{\mathrm{Cq}-\mathrm{ROF}}^{\mathrm{G} 4}(B, A)$

3. $d_{\mathrm{Cq}-\mathrm{ROF}}^{\mathrm{G} 4}(A, B)=1$ if $A=B$.
If we consider the value of $\Upsilon=0$, then

$$
\begin{aligned}
& d_{\mathrm{C}-\mathrm{ROF}}^{\mathrm{G} 4}(A, B) \\
& =\left(\frac{\sum_{i=1}^{n}\left(\begin{array}{c}
\alpha_{A}^{q}\left(x_{i}\right) \alpha_{B}^{q}\left(x_{i}\right)+\beta_{A}^{q}\left(x_{i}\right) \beta_{B}^{q}\left(x_{i}\right)+\theta_{A}^{q}\left(x_{i}\right) \theta_{B}^{q}\left(x_{i}\right)+ \\
\delta_{\alpha_{A}}^{q}\left(x_{i}\right) \delta_{\alpha_{B}}^{q}\left(x_{i}\right)+\delta_{\beta_{A}}^{q}\left(x_{i}\right) \delta_{\beta_{B}}^{q}\left(x_{i}\right)+\delta_{\theta_{A}}^{q}\left(x_{i}\right) \delta_{\theta_{B}}^{q}\left(x_{i}\right)
\end{array}\right)}{\sum_{i=1}^{n}\left(\begin{array}{l}
\alpha_{B}^{2 q}\left(x_{i}\right)+\beta_{B}^{2 q}\left(x_{i}\right)+\theta_{B}^{2 q}\left(x_{i}\right)+ \\
\delta_{\alpha_{B}}^{2 q}\left(x_{i}\right)+\delta_{\beta_{B}}^{2 q}\left(x_{i}\right)+\delta_{\theta_{B}}^{2 q}\left(x_{i}\right)
\end{array}\right)}\right)
\end{aligned}
$$

If we consider the value of $\Upsilon=1$, then

$$
d_{\mathrm{Cq}-\mathrm{ROF}}^{\mathrm{G} 4}(A, B)
$$

$$
=\left(\frac{\sum_{i=1}^{n}\left(\begin{array}{c}
\alpha_{A}^{q}\left(x_{i}\right) \alpha_{B}^{q}\left(x_{i}\right)+\beta_{A}^{q}\left(x_{i}\right) \beta_{B}^{q}\left(x_{i}\right)+\theta_{A}^{q}\left(x_{i}\right) \theta_{B}^{q}\left(x_{i}\right)+ \\
\delta_{\alpha_{A}}\left(x_{i}\right) \delta_{\alpha_{B}}^{q}\left(x_{i}\right)+\delta_{\beta_{A}}^{q}\left(x_{i}\right) \delta_{\beta_{B}}^{q}\left(x_{i}\right)+\delta_{\theta_{A}}^{q}\left(x_{i}\right) \delta_{\theta_{B}}^{q}\left(x_{i}\right)
\end{array}\right)}{\sum_{i=1}^{n}\left(\begin{array}{l}
\alpha_{A}^{2 q}\left(x_{i}\right)+\beta_{A}^{2 q}\left(x_{i}\right)+\theta_{A}^{2 q}\left(x_{i}\right)+ \\
\delta_{\alpha_{A}}^{2 q}\left(x_{i}\right)+\delta_{\beta_{A}}^{2 q}\left(x_{i}\right)+\delta_{\theta_{A}}^{2 q}\left(x_{i}\right)
\end{array}\right)}\right)
$$

is called asymmetric similarity measures or projection similarity measures.

Definition 22 A WGDSM $d_{\mathrm{Cq}-\mathrm{ROF}}^{\mathrm{wG} 4}(A, B)$ for Cq-ROFSs is given by:

$d_{\mathrm{Cq}-\mathrm{ROF}}^{\mathrm{WG}}(A, B)$

$$
=\left(\begin{array}{c}
\sum_{i=1}^{n} \omega_{i}^{2 q}\left(\begin{array}{c}
\alpha_{A}^{q}\left(x_{i}\right) \alpha_{B}^{q}\left(x_{i}\right)+\beta_{A}^{q}\left(x_{i}\right) \beta_{B}^{q}\left(x_{i}\right)+\theta_{A}^{q}\left(x_{i}\right) \theta_{B}^{q}\left(x_{i}\right)+ \\
\delta_{\alpha_{A}}^{q}\left(x_{i}\right) \delta_{\alpha_{B}}^{q}\left(x_{i}\right)+\delta_{\beta_{A}}^{q}\left(x_{i}\right) \delta_{\beta_{B}}^{q}\left(x_{i}\right)+\delta_{\theta_{A}}^{q}\left(x_{i}\right) \delta_{\theta_{B}}^{q}\left(x_{i}\right)
\end{array}\right) \\
\Upsilon \sum_{i=1}^{n} \omega_{i}^{2 q}\left(\begin{array}{l}
\alpha_{A}^{2 q}\left(x_{i}\right)+\beta_{A}^{2 q}\left(x_{i}\right)+\theta_{A}^{2 q}\left(x_{i}\right)+ \\
\delta_{\alpha_{A}}^{2 q}\left(x_{i}\right)+\delta_{\beta_{A}}^{2 q}\left(x_{i}\right)+\delta_{\theta_{A}}^{2 q}\left(x_{i}\right)
\end{array}\right) \\
+(1-\Upsilon) \sum_{i=1}^{n} \omega_{i}^{2 q}\left(\begin{array}{l}
\alpha_{B}^{2 q}\left(x_{i}\right)+\beta_{B}^{2 q}\left(x_{i}\right)+\theta_{B}^{2 q}\left(x_{i}\right)+ \\
\delta_{\alpha_{B}}^{2 q}\left(x_{i}\right)+\delta_{\beta_{B}}^{2 q}\left(x_{i}\right)+\delta_{\theta_{B}}^{2 q}\left(x_{i}\right)
\end{array}\right)
\end{array}\right)
$$

which satisfy following conditions:

1. $0 \leq d_{\mathrm{Cq}-\mathrm{ROF}}^{\mathrm{CO}}(A, B) \leq 1$

2. $d_{\mathrm{Cq}-\mathrm{ROF}}^{\mathrm{wG} 4}(A, B)=d_{\mathrm{Cq}-\mathrm{ROF}}^{\mathrm{wG} 4}(B, A)$

3. $d_{\mathrm{Cq}-\mathrm{ROF}}^{\mathrm{wG} 4}(A, B)=1$ if $A=B$.

If we consider the value of $\Upsilon=0$, then

$$
\begin{aligned}
& d_{\mathrm{Cq}-\mathrm{ROF}}^{\mathrm{wG} 4}(A, B) \\
& =\left(\frac{\sum_{i=1}^{n} \omega_{i}^{2 q}\left(\begin{array}{c}
\alpha_{A}^{q}\left(x_{i}\right) \alpha_{B}^{q}\left(x_{i}\right)+\beta_{A}^{q}\left(x_{i}\right) \beta_{B}^{q}\left(x_{i}\right)+\theta_{A}^{q}\left(x_{i}\right) \theta_{B}^{q}\left(x_{i}\right)+ \\
\delta_{\alpha_{A}}^{q}\left(x_{i}\right) \delta_{\alpha_{B}}^{q}\left(x_{i}\right)+\delta_{\beta_{A}}^{q}\left(x_{i}\right) \delta_{\beta_{B}}^{q}\left(x_{i}\right)+\delta_{\theta_{A}}^{q}\left(x_{i}\right) \delta_{\theta_{B}}^{q}\left(x_{i}\right)
\end{array}\right)}{\sum_{i=1}^{n} \omega_{i}^{2 q}\left(\begin{array}{c}
\alpha_{B}^{2 q}\left(x_{i}\right)+\beta_{B}^{2 q}\left(x_{i}\right)+\theta_{B}^{2 q}\left(x_{i}\right) \\
+\delta_{\alpha_{B}}^{2 q}\left(x_{i}\right)+\delta_{\beta_{B}}^{2 q}\left(x_{i}\right)+\delta_{\theta_{B}}^{2 q}\left(x_{i}\right)
\end{array}\right)}\right)
\end{aligned}
$$

If we consider the value of $\Upsilon=1$, then 
Table 1 Representation of the certain diseases

\begin{tabular}{lllll}
\hline Symbol & $Q_{1}$ & $Q_{2}$ & $Q_{3}$ & $Q_{4}$ \\
\hline Representation & Viral fever & Malaria & Typhoid & Stomach issue \\
\hline
\end{tabular}

Table 2 Representations of symptoms

\begin{tabular}{lllll}
\hline Symbols & $s_{1}$ & $s_{2}$ & $s_{3}$ & $s_{4}$ \\
\hline Representations & Temperature & Headache & $\begin{array}{c}\text { Stomach } \\
\text { Pain }\end{array}$ & Cough \\
\hline
\end{tabular}

$$
\begin{aligned}
& d_{\mathrm{Cq}-\mathrm{ROF}}^{\mathrm{wG} 4}(A, B) \\
& =\left(\frac{\sum_{i=1}^{n} \omega_{i}^{2 q}\left(\begin{array}{c}
\alpha_{A}^{q}\left(x_{i}\right) \alpha_{B}^{q}\left(x_{i}\right)+\beta_{A}^{q}\left(x_{i}\right) \beta_{B}^{q}\left(x_{i}\right)+\theta_{A}^{q}\left(x_{i}\right) \theta_{B}^{q}\left(x_{i}\right)+ \\
\delta_{\alpha_{A}}^{q}\left(x_{i}\right) \delta_{\alpha_{B}}^{q}\left(x_{i}\right)+\delta_{\beta_{A}}^{q}\left(x_{i}\right) \delta_{\beta_{B}}^{q}\left(x_{i}\right)+\delta_{\theta_{A}}^{q}\left(x_{i}\right) \delta_{\theta_{B}}^{q}\left(x_{i}\right)
\end{array}\right)}{\sum_{i=1}^{n} \omega_{i}^{2 q}\left(\begin{array}{l}
\alpha_{A}^{2 q}\left(x_{i}\right)+\beta_{A}^{2 q}\left(x_{i}\right)+\theta_{A}^{2 q}\left(x_{i}\right)+ \\
\delta_{\alpha_{A}}^{2 q}\left(x_{i}\right)+\delta_{\beta_{A}}^{2 q}\left(x_{i}\right)+\delta_{\theta_{A}}^{2 q}\left(x_{i}\right)
\end{array}\right)}\right)
\end{aligned}
$$

is called asymmetric similarity measures or projection similarity measures.

Remark 18 By changing $\Upsilon=0.5$, the Eqs. (46) and (49) are reduced into Eqs. (18) and (19).

Remark 19 If we take $\omega=\left(\frac{1}{n}, \frac{1}{n}, \ldots, \frac{1}{n}\right)^{T}$ then the WGDSM for Cq-ROFS reduced to GDSM for Cq-ROFS i.e. $d_{\mathrm{Cq}-\mathrm{ROF}}^{\mathrm{wG} 4}(A, B)=d_{\mathrm{Cq}-\mathrm{ROF}}^{\mathrm{G} 4}(A, B)$.

Remark 20 The GDDM and WGDDM for Cq-ROFSs are defined as:

$$
\begin{aligned}
& d d_{\mathrm{Cq}-\mathrm{ROF}}^{\mathrm{G} 4}(A, B)=1-d_{\mathrm{Cq}-\mathrm{ROF}}^{\mathrm{G} 4}(A, B) \\
& d d_{\mathrm{Cq}-\mathrm{ROF}}^{\mathrm{wG} 4}(A, B)=1-d_{\mathrm{Cq}-\mathrm{ROF}}^{\mathrm{wG} 4}(A, B)
\end{aligned}
$$

\section{Illustrative examples}

In order to illustrate the validity and feasibility of the proposed approaches, we discussed some examples in the areas of medical diagnosis and pattern recognition. The Examples 1,3 and 5 are discussed for medical diagnosis while the Examples 2, 4 and 6 are discussed for patterns recognitions.

Example 1 In this example, we consider the set of diseases, denoted by $Q_{1}, Q_{2}, Q_{3}$ and $Q_{4}$ whose details is represented in Table 1 and set of symptoms which are described in Table 2.

Consider the patient $(\mathrm{P})$ which are evaluated with respect to all the symptoms by an expert and represent their rating in terms of Cq-ROFNs given in Table 3.
On the other hand, experts also provide information related to each diseases $Q_{i}(i=1,2,3,4)$ with respect to each symptoms and their rating are described in Table 4. Let $\omega=(0.23,0.27,0.22,0.28)$ be the weight vector of the symptoms.

Next, we will examine diagnosis of the patient $P$ with respect to diseases $Q_{i}$ by using Eq. (49), with $q=3$ and we can get

$d_{\mathrm{Cq}-\mathrm{ROF}}^{\mathrm{wG} 4}\left(Q_{1}, P\right)=0.02, d_{\mathrm{Cq}-\mathrm{ROF}}^{\mathrm{wG} 4}\left(Q_{2}, P\right)=0.15$,
$d_{\mathrm{Cq}-\mathrm{ROF}}^{\mathrm{wG} 4}\left(Q_{3}, P\right)=0.009, d_{\mathrm{Cq}-\mathrm{ROF}}^{\mathrm{wG} 4}\left(Q_{4}, P\right)=0.01$

The ranking of the values is follow as:

$Q_{3} \leq Q_{4} \leq Q_{2} \leq Q_{1}$

Thus, on the basis on ranking methods, we conclude that the patient $P$ suffers from the diseases $Q_{1}$. In order to examine the effectiveness and dominance of the investigated approach based on dice similarity measures, we conducted a comparison between proposed approach and existing methods proposed by Garg and Rani [36], Rani and Garg [39] and Ullah et al. [41]. For this, we used the proposed approach in Eq. (49) and their corresponding results are described in Table 5, which express the patient $P$ suffers from the diseases $Q_{i}$.

Example 2 Consider the four known patterns, which are denoted the Cq-ROFNs is described in Table 6. Suppose the unknown pattern is represented by the following Cq-ROFNs in Table 7.

Next, we will examine similarity between unknown pattern $B$ with respect to known patterns $A_{i}$ by using Eq. (46) with $q=3$ and we can get.

$d_{\mathrm{Cq}-\mathrm{ROF}}^{\mathrm{G} 4}\left(A_{1}, B\right)=0.015, \quad d_{\mathrm{Cq}-\mathrm{ROF}}^{\mathrm{G} 4}\left(A_{2}, B\right)=0.012$,

$d_{\mathrm{Cq}-\mathrm{ROF}}^{\mathrm{G} 4}\left(A_{3}, B\right)=0.007, \quad d_{\mathrm{Cq}-\mathrm{ROF}}^{\mathrm{G} 4}\left(A_{4}, B\right)=0.009$

The ranking of the values is follow as:

$A_{3} \leq A_{4} \leq A_{2} \leq A_{1}$

Thus, on the based on ranking methods, we conclude that the unknown patterns $P$ belong to the pattern $A_{1}$. In order to examine the effectiveness and dominance of the investigated approach based on dice similarity measures, we conducted a comparison between proposed approach and existing methods proposed by Garg and Rani [36], Rani and Garg [39] and Ullah et al. [41]. For this, we used the proposed approach in Eq. (46) and their corresponding results are described in Table 8. 
Table 3 Decision matrix for patient with respect to symptoms

Table 4 Decision metric of complex q-rung orthopair fuzzy sets

Table 5 Comparison between proposed methods with existing methods

\begin{tabular}{lllll}
\hline Symptoms & $s_{1}$ & $s_{2}$ & $s_{3}$ & $s_{4}$ \\
\hline Patient & $\left(\begin{array}{l}(0.5) e^{i 2 \pi(0.45)}, \\
(0.4) e^{i 2 \pi(0.44)}\end{array}\right)$ & $\left(\begin{array}{l}(0.51) e^{i 2 \pi(0.46)}, \\
(0.39) e^{i 2 \pi(0.43)}\end{array}\right)$ & $\left(\begin{array}{l}(0.52) e^{i 2 \pi(0.47)}, \\
(0.38) e^{i 2 \pi(0.42)}\end{array}\right)$ & $\left(\begin{array}{l}(0.53) e^{i 2 \pi(0.48)}, \\
(0.37) e^{i 2 \pi(0.41)}\end{array}\right)$ \\
\hline
\end{tabular}

\begin{tabular}{lllll}
\hline & $Q_{1}$ & $Q_{2}$ & $Q_{3}$ & $Q_{4}$ \\
\hline$s_{1}$ & $\left(\begin{array}{l}(0.51) e^{i 2 \pi(0.41)}, \\
(0.41) e^{i 2 \pi(0.41)}\end{array}\right)$ & $\left(\begin{array}{l}(0.52) e^{i 2 \pi(0.34)}, \\
(0.32) e^{i 2 \pi(0.33)}\end{array}\right)$ & $\left(\begin{array}{l}(0.22) e^{i 2 \pi(0.44)}, \\
(0.28) e^{i 2 \pi(0.4)}\end{array}\right)$ & $\left(\begin{array}{l}(0.47) e^{i 2 \pi(0.42)}, \\
(0.33) e^{i 2 \pi(0.31)}\end{array}\right)$ \\
$s_{2}$ & $\left(\begin{array}{l}(0.52) e^{i 2 \pi(0.42)}, \\
(0.42) e^{i 2 \pi(0.42)}\end{array}\right)$ & $\left(\begin{array}{l}(0.53) e^{i 2 \pi(0.36)}, \\
(0.33) e^{i 2 \pi(0.23)}\end{array}\right)$ & $\left(\begin{array}{l}(0.32) e^{i 2 \pi(0.41)}, \\
(0.18) e^{i 2 \pi(0.34)}\end{array}\right)$ & $\left(\begin{array}{l}(0.43) e^{i 2 \pi(0.39)}, \\
(0.35) e^{i 2 \pi(0.4)}\end{array}\right)$ \\
$s_{3}$ & $\left(\begin{array}{l}(0.53) e^{i 2 \pi(0.43)}, \\
(0.43) e^{i 2 \pi(0.43)}\end{array}\right)$ & $\left(\begin{array}{l}(0.54) e^{i 2 \pi(0.36)}, \\
(0.34) e^{i 2 \pi(0.43)}\end{array}\right)$ & $\left(\begin{array}{l}(0.52) e^{i 2 \pi(0.34)}, \\
(0.18) e^{i 2 \pi(0.45)}\end{array}\right)$ & $\left(\begin{array}{l}(0.33) e^{i 2 \pi(0.48)}, \\
(0.32) e^{i 2 \pi(0.21)}\end{array}\right)$ \\
$s_{4}$ & $\left(\begin{array}{l}(0.54) e^{i 2 \pi(0.44)}, \\
(0.44) e^{i 2 \pi(0.44)}\end{array}\right)$ & $\left(\begin{array}{l}(0.55) e^{i 2 \pi(0.16)}, \\
(0.35) e^{i 2 \pi(0.23)}\end{array}\right)$ & $\left(\begin{array}{l}(0.32) e^{i 2 \pi(0.23)}, \\
(0.33) e^{i 2 \pi(0.32)}\end{array}\right)$ & $\left(\begin{array}{l}(0.43) e^{i 2 \pi(0.14)}, \\
(0.34) e^{i 2 \pi(0.39)}\end{array}\right)$
\end{tabular}

\begin{tabular}{|c|c|c|}
\hline Methods & Values of examined by methods & Ranking \\
\hline Garg and Rani [36] & $\begin{array}{l}d_{\mathrm{Cq}-\mathrm{ROF}}^{\mathrm{wG} 4}\left(Q_{1}, P\right)=0.2, \\
d_{\mathrm{Cq}-\mathrm{ROF}}^{\mathrm{wG}}\left(Q_{2}, P\right)=0.15, \\
d_{\mathrm{Cq}-\mathrm{ROF}}^{\mathrm{wG}}\left(Q_{3}, P\right)=0.009, \\
d_{\mathrm{Cq}-\mathrm{ROF}}^{\mathrm{wG}}\left(Q_{4}, P\right)=0.11\end{array}$ & $Q_{1} \leq Q_{2} \leq Q_{3} \leq Q_{4}$ \\
\hline Rani and Garg [39] & $\begin{array}{l}d_{\mathrm{Cq}-\mathrm{ROF}}^{\mathrm{wG}}\left(Q_{1}, P\right)=0.99, \\
d^{\mathrm{wG} 4} \quad\left(Q_{2}, P\right)=0.88, \\
d_{\mathrm{Cq}-\mathrm{ROF}-\mathrm{ROF}}^{\mathrm{wGOF}}\left(Q_{3}, P\right)=0.81, \\
d_{\mathrm{Cq}-\mathrm{ROF}}^{\mathrm{wG}}\left(Q_{4}, P\right)=0.86\end{array}$ & $Q_{3} \leq Q_{4} \leq Q_{2} \leq Q_{1}$ \\
\hline Ullah et al. [41] & $\begin{array}{l}d_{\mathrm{Cq}-\mathrm{ROF}}^{\mathrm{wG} 4}\left(Q_{1}, P\right)=0.14 \\
d_{\mathrm{Cq}-\mathrm{ROF}}^{\mathrm{wG}}\left(Q_{2}, P\right)=0.10 \\
d_{\mathrm{Cq}-\mathrm{ROF}}^{\mathrm{wG}}\left(Q_{3}, P\right)=0.07 \\
d_{\mathrm{Cq}-\mathrm{ROF}}^{\mathrm{wG}}\left(Q_{4}, P\right)=0.09\end{array}$ & $Q_{3} \leq Q_{4} \leq Q_{2} \leq Q_{1}$ \\
\hline Proposed methods for $q=1$ & $\begin{array}{l}d_{\mathrm{Cq}-\mathrm{ROF}}^{\mathrm{wG} 4}\left(Q_{1}, P\right)=0.99, \\
d_{\mathrm{Cq}-\mathrm{ROF}}^{\mathrm{wG}}\left(Q_{2}, P\right)=0.88, \\
d_{\mathrm{Cq}-\mathrm{ROF}}^{\mathrm{wG}}\left(Q_{3}, P\right)=0.81, \\
d_{\mathrm{Cq}-\mathrm{ROF}}^{\mathrm{wG}}\left(Q_{4}, P\right)=0.87\end{array}$ & $Q_{3} \leq Q_{4} \leq Q_{2} \leq Q_{1}$ \\
\hline Proposed methods for $q=2$ & $\begin{array}{l}d_{\mathrm{Cq}-\mathrm{ROF}}^{\mathrm{wG} 4}\left(Q_{1}, P\right)=0.14, \\
d_{\mathrm{Cq}-\mathrm{ROF}}^{\mathrm{wG}}\left(Q_{2}, P\right)=0.10, \\
d_{\mathrm{Cq}-\mathrm{ROF}}^{\mathrm{wG}}\left(Q_{3}, P\right)=0.08, \\
d_{\mathrm{Cq}-\mathrm{ROF}}^{\mathrm{wG}}\left(Q_{4}, P\right)=0.09\end{array}$ & $Q_{3} \leq Q_{4} \leq Q_{2} \leq Q_{1}$ \\
\hline Proposed methods for $q=3$ & $\begin{array}{l}d_{\mathrm{Cq}-\mathrm{ROF}}^{\mathrm{wG} 4}\left(Q_{1}, P\right)=0.02, \\
d_{\mathrm{Cq}-\mathrm{ROF}}^{\mathrm{wG}}\left(Q_{2}, P\right)=0.15, \\
d_{\mathrm{Cq}-\mathrm{ROF}}^{\mathrm{wG}}\left(Q_{3}, P\right)=0.009, \\
d_{\mathrm{Cq}-\mathrm{ROF}}^{\mathrm{wG} 4}\left(Q_{4}, P\right)=0.01\end{array}$ & $Q_{3} \leq Q_{4} \leq Q_{2} \leq Q_{1}$ \\
\hline
\end{tabular}

The example of pattern recognition is solved for without weight vector and compared the results with other existing methods. The advantages and comparative analysis of the proposed methods are discussed below.

\section{Comparative analysis}

In this sub-section, we compared our proposed approaches with existing approaches to examine the stability and effectiveness of the proposed methods. We will compare the existing methods with proposed methods by numerical exam- 
Table 6 Decision matrix for known patterns

Table 7 Decision matrix for unknown pattern

Table 8 Comparison between proposed methods with existing methods

\begin{tabular}{lllll}
\hline Symbols & $A_{1}$ & $A_{2}$ & $A_{3}$ & $A_{4}$ \\
\hline$x_{1}$ & $\left(\begin{array}{l}(0.51) e^{i 2 \pi(0.41)}, \\
(0.41) e^{i 2 \pi(0.41)}\end{array}\right)$ & $\left(\begin{array}{l}(0.51) e^{i 2 \pi(0.41)}, \\
(0.41) e^{i 2 \pi(0.41)}\end{array}\right)$ & $\left(\begin{array}{l}(0.51) e^{i 2 \pi(0.41)}, \\
(0.41) e^{i 2 \pi(0.41)}\end{array}\right)$ & $\left(\begin{array}{l}(0.51) e^{i 2 \pi(0.41)}, \\
(0.41) e^{i 2 \pi(0.41)}\end{array}\right)$ \\
$x_{2}$ & $\left(\begin{array}{l}(0.52) e^{i 2 \pi(0.42)}, \\
(0.42) e^{i 2 \pi(0.42)}\end{array}\right)$ & $\left(\begin{array}{l}(0.52) e^{i 2 \pi(0.42)}, \\
(0.42) e^{i 2 \pi(0.42)}\end{array}\right)$ & $\left(\begin{array}{l}(0.52) e^{i 2 \pi(0.42)}, \\
(0.42) e^{i 2 \pi(0.42)}\end{array}\right)$ & $\left(\begin{array}{l}(0.52) e^{i 2 \pi(0.42)}, \\
(0.42) e^{i 2 \pi(0.42)}\end{array}\right)$ \\
$x_{3}$ & $\left(\begin{array}{l}(0.53) e^{i 2 \pi(0.43)}, \\
(0.43) e^{i 2 \pi(0.43)}\end{array}\right)$ & $\left(\begin{array}{l}(0.53) e^{i 2 \pi(0.43)}, \\
(0.43) e^{i 2 \pi(0.43)}\end{array}\right)$ & $\left(\begin{array}{l}(0.53) e^{i 2 \pi(0.43)}, \\
(0.43) e^{i 2 \pi(0.43)}\end{array}\right)$ & $\left(\begin{array}{l}(0.53) e^{i 2 \pi(0.43)}, \\
(0.43) e^{i 2 \pi(0.43)}\end{array}\right)$ \\
$x_{4}$ & $\left(\begin{array}{l}(0.54) e^{i 2 \pi(0.44)}, \\
(0.44) e^{i 2 \pi(0.44)}\end{array}\right)$ & $\left(\begin{array}{l}(0.54) e^{i 2 \pi(0.44)}, \\
(0.44) e^{i 2 \pi(0.44)}\end{array}\right)$ & $\left(\begin{array}{l}(0.54) e^{i 2 \pi(0.44)}, \\
(0.44) e^{i 2 \pi(0.44)}\end{array}\right)$ & $\left(\begin{array}{l}(0.54) e^{i 2 \pi(0.44)}, \\
(0.44) e^{i 2 \pi(0.44)}\end{array}\right)$ \\
\hline
\end{tabular}

\begin{tabular}{lllll}
\hline Symbols & $x_{1}$ & $x_{2}$ & $x_{3}$ & $x_{4}$ \\
\hline$B$ & $\left(\begin{array}{l}(0.5) e^{i 2 \pi(0.45)}, \\
(0.4) e^{i 2 \pi(0.44)}\end{array}\right)$ & $\left(\begin{array}{l}(0.51) e^{i 2 \pi(0.46)}, \\
(0.39) e^{i 2 \pi(0.43)}\end{array}\right)$ & $\left(\begin{array}{l}(0.52) e^{i 2 \pi(0.47)}, \\
(0.38) e^{i 2 \pi(0.42)}\end{array}\right)$ & $\left(\begin{array}{l}(0.53) e^{i 2 \pi(0.48)}, \\
(0.37) e^{i 2 \pi(0.41)}\end{array}\right)$ \\
\hline
\end{tabular}

\begin{tabular}{|c|c|c|}
\hline Methods & Values of examined by methods & Ranking \\
\hline Garg and Rani [36] & $\begin{array}{l}d_{\mathrm{Cq}-\mathrm{ROF}}^{\mathrm{G} 4}\left(A_{1}, B\right)=0.015, \\
d_{\mathrm{Cq}-\mathrm{ROF}}^{\mathrm{G} 4}\left(A_{2}, B\right)=0.012, \\
d_{\mathrm{Cq}-\mathrm{ROF}}^{\mathrm{G} 4}\left(A_{3}, B\right)=0.008, \\
d_{\mathrm{Cq}-\mathrm{ROF}}^{\mathrm{G} 4}\left(A_{4}, B\right)=0.009\end{array}$ & $A_{1} \leq A_{2} \leq A_{3} \leq A_{4}$ \\
\hline Rani and Garg [39] & $\begin{array}{l}d_{\mathrm{Cq}-\mathrm{ROF}}^{\mathrm{G} 4}\left(A_{1}, B\right)=0.99, \\
d_{\mathrm{Cq}-\mathrm{ROF}}^{\mathrm{G} 4}\left(A_{2}, B\right)=0.89, \\
d_{\mathrm{Cq}-\mathrm{ROF}}^{\mathrm{G}}\left(A_{3}, B\right)=0.82, \\
d_{\mathrm{Cq}-\mathrm{ROF}}^{\mathrm{G} 4}\left(A_{4}, B\right)=0.86\end{array}$ & $A_{3} \leq A_{4} \leq A_{2} \leq A_{1}$ \\
\hline Ullah et al. [41] & $\begin{array}{l}d_{\mathrm{Cq}-\mathrm{ROF}}^{\mathrm{G} 4}\left(A_{1}, B\right)=0.10, \\
d_{\mathrm{Cq}-\mathrm{ROF}}^{\mathrm{G} 4}\left(A_{2}, B\right)=0.08, \\
d_{\mathrm{Cq}-\mathrm{ROF}}^{\mathrm{G}}\left(A_{3}, B\right)=0.06, \\
d_{\mathrm{Cq}-\mathrm{ROF}}^{\mathrm{G} 4}\left(A_{4}, B\right)=0.07\end{array}$ & $A_{3} \leq A_{4} \leq A_{2} \leq A_{1}$ \\
\hline Proposed methods for $q=1$ & $\begin{array}{l}d_{\mathrm{Cq}-\mathrm{ROF}}^{\mathrm{G} 4}\left(A_{1}, B\right)=0.99, \\
d_{\mathrm{Cq}-\mathrm{ROF}}^{\mathrm{G} 4}\left(A_{2}, B\right)=0.88, \\
d_{\mathrm{Cq}-\mathrm{ROF}}^{\mathrm{G}}\left(A_{3}, B\right)=0.82 \\
d_{\mathrm{Cq}-\mathrm{ROF}}^{\mathrm{G} 4}\left(A_{4}, B\right)=0.87\end{array}$ & $A_{3} \leq A_{4} \leq A_{2} \leq A_{1}$ \\
\hline Proposed methods for $q=2$ & $\begin{array}{l}d_{\mathrm{Cq}-\mathrm{ROF}}^{\mathrm{G} 4}\left(A_{1}, B\right)=0.10, \\
d_{\mathrm{Cq}-\mathrm{ROF}}^{\mathrm{G} 4}\left(A_{2}, B\right)=0.081, \\
d_{\mathrm{Cq}-\mathrm{ROF}}^{\mathrm{G} 4}\left(A_{3}, B\right)=0.062, \\
d_{\mathrm{Cq}-\mathrm{ROF}}^{\mathrm{G} 4}\left(A_{4}, B\right)=0.072\end{array}$ & $A_{3} \leq A_{4} \leq A_{2} \leq A_{1}$ \\
\hline Proposed methods for $q=3$ & $\begin{aligned} d_{\mathrm{Cq}-\mathrm{ROF}}^{\mathrm{G} 4}\left(A_{1}, B\right) & =0.015, \\
d_{\mathrm{Cq}-\mathrm{ROF}}^{\mathrm{G} 4}\left(A_{2}, B\right) & =0.012, \\
d_{\mathrm{Cq}-\mathrm{ROF}}^{\mathrm{G}}\left(A_{3}, B\right) & =0.007, \\
d_{\mathrm{Cq}-\mathrm{ROF}}^{\mathrm{G} 4}\left(A_{4}, B\right) & =0.009\end{aligned}$ & $A_{3} \leq A_{4} \leq A_{2} \leq A_{1}$ \\
\hline
\end{tabular}

ples. The proposed methods are compared with Garg and Rani [36], Rani and Garg [39] and Ullah et al. [41].

Example 3 Consider, the patient with respect to all the symptoms can be represented by the following Cq-ROFNs in Table 9.
And each diseases $Q_{i}(i=1,2,3,4)$ is described in Table 10 with respect to weight vector $\omega=$ $(0.23,0.27,0.22,0.28)$.

Next, we will examine diagnosis of the patient $P$ with respect to diseases $Q_{i}$ by using Eq. (49), with $q=3$ and we can get 

patient with respect to symptoms

Table 10 Decision matrix of complex q-rung orthopair fuzzy sets
Table 9 Decision matrix for

\begin{tabular}{lllll}
\hline Symbols & $s_{1}$ & $s_{2}$ & $s_{3}$ & $s_{4}$ \\
\hline Patient (P) & $\left(\begin{array}{l}(0.7) e^{i 2 \pi(0.55)}, \\
(0.4) e^{i 2 \pi(0.44)}\end{array}\right)$ & $\left(\begin{array}{l}(0.71) e^{i 2 \pi(0.56)}, \\
(0.39) e^{i 2 \pi(0.43)}\end{array}\right)$ & $\left(\begin{array}{l}(0.72) e^{i 2 \pi(0.57)}, \\
(0.38) e^{i 2 \pi(0.42)}\end{array}\right)$ & $\left(\begin{array}{l}(0.73) e^{i 2 \pi(0.58)}, \\
(0.37) e^{i 2 \pi(0.41)}\end{array}\right)$ \\
\hline
\end{tabular}

\begin{tabular}{lllll}
\hline Symbols & $Q_{1}$ & $Q_{2}$ & $Q_{3}$ & $Q_{4}$ \\
\hline$s_{1}$ & $\left(\begin{array}{l}(0.71) e^{i 2 \pi(0.51)}, \\
(0.41) e^{i 2 \pi(0.41)}\end{array}\right)$ & $\left(\begin{array}{l}(0.72) e^{i 2 \pi(0.44)}, \\
(0.32) e^{i 2 \pi(0.33)}\end{array}\right)$ & $\left(\begin{array}{l}(0.42) e^{i 2 \pi(0.54)}, \\
(0.28) e^{i 2 \pi(0.4)}\end{array}\right)$ & $\left(\begin{array}{l}(0.67) e^{i 2 \pi(0.52)}, \\
(0.33) e^{i 2 \pi(0.31)}\end{array}\right)$ \\
$s_{2}$ & $\left(\begin{array}{l}(0.72) e^{i 2 \pi(0.52)}, \\
(0.42) e^{i 2 \pi(0.42)}\end{array}\right)$ & $\left(\begin{array}{l}(0.73) e^{i 2 \pi(0.46)}, \\
(0.33) e^{i 2 \pi(0.23)}\end{array}\right)$ & $\left(\begin{array}{l}(0.52) e^{i 2 \pi(0.51)}, \\
(0.18) e^{i 2 \pi(0.34)}\end{array}\right)$ & $\left(\begin{array}{l}(0.63) e^{i 2 \pi(0.49)}, \\
(0.35) e^{i 2 \pi(0.4)}\end{array}\right)$ \\
$s_{3}$ & $\left(\begin{array}{l}(0.73) e^{i 2 \pi(0.53)}, \\
(0.43) e^{i 2 \pi(0.43)}\end{array}\right)$ & $\left(\begin{array}{l}(0.74) e^{i 2 \pi(0.46)}, \\
(0.34) e^{i 2 \pi(0.43)}\end{array}\right)$ & $\left(\begin{array}{l}(0.72) e^{i 2 \pi(0.44)}, \\
(0.18) e^{i 2 \pi(0.45)}\end{array}\right)$ & $\left(\begin{array}{l}(0.53) e^{i 2 \pi(0.58)}, \\
(0.32) e^{i 2 \pi(0.21)}\end{array}\right)$ \\
$s_{4}$ & $\left(\begin{array}{l}(0.74) e^{i 2 \pi(0.54)}, \\
(0.44) e^{i 2 \pi(0.44)}\end{array}\right)$ & $\left(\begin{array}{l}(0.75) e^{i 2 \pi(0.26)}, \\
(0.35) e^{i 2 \pi(0.23)}\end{array}\right)$ & $\left(\begin{array}{l}(0.52) e^{i 2 \pi(0.33)}, \\
(0.33) e^{i 2 \pi(0.32)}\end{array}\right)$ & $\left(\begin{array}{l}(0.63) e^{i 2 \pi(0.24)}, \\
(0.34) e^{i 2 \pi(0.39)}\end{array}\right)$ \\
\hline
\end{tabular}

Thus, on the based on ranking methods, we conclude that the Unknown patterns $P$ belong to the pattern $A_{i}$. In order to examine the effectiveness and dominance of the investigated approach based on dice similarity measures, we conducted a comparison between proposed approach and existing methods proposed by Garg and Rani [36], Rani and Garg [39] and Ullah et al. [41]. For this, we used the proposed approach in Eq. (46) and their corresponding results are described in Table 14, which express the unknown pattern $P$ belonging to the pattern $A_{i}$.

Example 5 Consider, the patient with respect to all the symptoms can be represented by the Cq-ROFNs in Table 15 .

And each diseases $Q_{i}(i=1,2,3,4)$ is described in Table 16 with respect to weight vector $\omega=$ $(0.23,0.27,0.22,0.28)$.

Next, we will examine diagnosis of the patient $P$ with respect to diseases $Q_{i}$ by using Eq. (49) with $q=5$, then we get

$d_{\mathrm{Cq}-\mathrm{ROF}}^{\mathrm{wG} 4}\left(Q_{1}, P\right)=0.04, \quad d_{\mathrm{Cq}-\mathrm{ROF}}^{\mathrm{wG} 4}\left(Q_{2}, P\right)=0.01$,

$d_{\mathrm{Cq}-\mathrm{ROF}}^{\mathrm{wG} 4}\left(Q_{3}, P\right)=0.02, \quad d_{\mathrm{Cq}-\mathrm{ROF}}^{\mathrm{wG} 4}\left(Q_{4}, P\right)=0.03$

The ranking of the values is follow as:

Suppose the unknown pattern is represented by the CqROFNs described in Table 13.

Next, we will examine similarity between unknown pattern $B$ with respect to known patterns $A_{i}$ by using Eq. (46) we get.

$d_{\mathrm{Cq}-\mathrm{ROF}}^{\mathrm{G} 4}\left(A_{1}, B\right)=0.18, \quad d_{\mathrm{Cq}-\mathrm{ROF}}^{\mathrm{G} 4}\left(A_{2}, B\right)=0.16$,

$d_{\mathrm{Cq}-\mathrm{ROF}}^{\mathrm{G} 4}\left(A_{3}, B\right)=0.12, \quad d_{\mathrm{Cq}-\mathrm{ROF}}^{\mathrm{G} 4}\left(A_{4}, B\right)=0.14$

The ranking of the values is follow as:

$A_{3} \leq A_{4} \leq A_{2} \leq A_{1}$
$Q_{2} \leq Q_{3} \leq Q_{4} \leq Q_{1}$

Thus, on the based on ranking methods, we conclude that the patient $P$ suffers from the diseases $Q_{1}$. In order to examine the effectiveness and dominance of the investigated approach based on dice similarity measures, we conducted a comparison between proposed approach and existing methods proposed by Garg and Rani [36], Rani and Garg [39] and Ullah et al. [41]. For this, we used the proposed approach in Eq. (49) and their corresponding results are described in Table 17, which express the patient $P$ suffers from the diseases $Q_{1}$. 
Table 11 Comparison between proposed methods with existing methods

Table 12 Decision matrix for known patterns

Table 13 Decision matrix for unknown patterns

Table 14 Comparison between proposed methods with existing methods

\begin{tabular}{lll}
\hline Methods & Values of examined by methods & Ranking \\
\hline Garg and Rani [36] & Cannot be calculated & Cannot be calculated \\
Rani and Garg [39] & Cannot be calculated & Cannot be calculated \\
Ullah et al. [41] & $d_{\mathrm{Cq}-\mathrm{ROF}}^{\mathrm{wG} 4}\left(Q_{1}, P\right)=0.20$, & $Q_{3} \leq Q_{4} \leq Q_{1} \leq Q_{2}$ \\
& $d_{\mathrm{Cq}-\mathrm{ROF}}^{\mathrm{wG}}\left(Q_{2}, P\right)=0.23$, & \\
& $d_{\mathrm{Cq}-\mathrm{ROF}}^{\mathrm{wG}}\left(Q_{3}, P\right)=0.14$, & \\
& $d_{\mathrm{Cq}-\mathrm{ROF}}^{\mathrm{wG}}\left(Q_{4}, P\right)=0.17$ & \\
Proposed methods for $q=2$ & $d_{\mathrm{Cq}-\mathrm{ROF}}^{\mathrm{wG} 4}\left(Q_{1}, P\right)=0.217$, & \\
& $d_{\mathrm{Cq}-\mathrm{ROF}}^{\mathrm{wG} 4}\left(Q_{2}, P\right)=0.224$, & \\
& $d_{\mathrm{Cq}-\mathrm{ROF}}^{\mathrm{wG}}\left(Q_{3}, P\right)=0.146$, & \\
& $d_{\mathrm{Cq}-\mathrm{ROF}}^{\mathrm{wG} 4}\left(Q_{4}, P\right)=0.167$ & \\
& $d_{\mathrm{Cq}-\mathrm{ROF}}^{\mathrm{wG}}\left(Q_{1}, P\right)=0.06$, & \\
& $d_{\mathrm{Cq}-\mathrm{ROF}}^{\mathrm{wG} 4}\left(Q_{2}, P\right)=0.07$, & \\
& $d_{\mathrm{Cq}-\mathrm{ROF}}^{\mathrm{wG}}\left(Q_{3}, P\right)=0.004$, & \\
& $d_{\mathrm{Cq}-\mathrm{ROF}}^{\mathrm{wG} 4}\left(Q_{4}, P\right)=0.005$ & \\
\hline
\end{tabular}

\begin{tabular}{lllll}
\hline Symbols & $A_{1}$ & $A_{2}$ & $A_{3}$ & $A_{4}$ \\
\hline$x_{1}$ & $\left(\begin{array}{l}(0.71) e^{i 2 \pi(0.51)}, \\
(0.41) e^{i 2 \pi(0.41)}\end{array}\right)$ & $\left(\begin{array}{l}(0.72) e^{i 2 \pi(0.44)}, \\
(0.32) e^{i 2 \pi(0.33)}\end{array}\right)$ & $\left(\begin{array}{l}(0.42) e^{i 2 \pi(0.54)}, \\
(0.28) e^{i 2 \pi(0.4)}\end{array}\right)$ & $\left(\begin{array}{l}(0.67) e^{i 2 \pi(0.52)}, \\
(0.33) e^{i 2 \pi(0.31)}\end{array}\right)$ \\
$x_{2}$ & $\left(\begin{array}{l}(0.72) e^{i 2 \pi(0.52)}, \\
(0.42) e^{i 2 \pi(0.42)}\end{array}\right)$ & $\left(\begin{array}{l}(0.73) e^{i 2 \pi(0.46)}, \\
(0.33) e^{i 2 \pi(0.23)}\end{array}\right)$ & $\left(\begin{array}{l}(0.52) e^{i 2 \pi(0.51)}, \\
(0.18) e^{i 2 \pi(0.34)}\end{array}\right)$ & $\left(\begin{array}{l}(0.63) e^{i 2 \pi(0.49)}, \\
(0.35) e^{i 2 \pi(0.4)}\end{array}\right)$ \\
$x_{3}$ & $\left(\begin{array}{l}(0.73) e^{i 2 \pi(0.53)}, \\
(0.43) e^{i 2 \pi(0.43)}\end{array}\right)$ & $\left(\begin{array}{l}(0.74) e^{i 2 \pi(0.46)}, \\
(0.34) e^{i 2 \pi(0.43)}\end{array}\right)$ & $\left(\begin{array}{l}(0.72) e^{i 2 \pi(0.44)}, \\
(0.18) e^{i 2 \pi(0.45)}\end{array}\right)$ & $\left(\begin{array}{l}(0.53) e^{i 2 \pi(0.58)}, \\
(0.32) e^{i 2 \pi(0.21)}\end{array}\right)$ \\
$x_{4}$ & $\left(\begin{array}{l}(0.74) e^{i 2 \pi(0.54)}, \\
(0.44) e^{i 2 \pi(0.44)}\end{array}\right)$ & $\left(\begin{array}{l}(0.75) e^{i 2 \pi(0.26)}, \\
(0.35) e^{i 2 \pi(0.23)}\end{array}\right)$ & $\left(\begin{array}{l}(0.52) e^{i 2 \pi(0.33)}, \\
(0.33) e^{i 2 \pi(0.32)}\end{array}\right)$ & $\left(\begin{array}{l}(0.63) e^{i 2 \pi(0.24)}, \\
(0.34) e^{i 2 \pi(0.39)}\end{array}\right)$ \\
\hline
\end{tabular}

\begin{tabular}{lllll}
\hline Symbols & $x_{1}$ & $x_{2}$ & $x_{3}$ & $x_{4}$ \\
\hline$B$ & $\left(\begin{array}{l}(0.7) e^{i 2 \pi(0.55)}, \\
(0.4) e^{i 2 \pi(0.44)}\end{array}\right)$ & $\left(\begin{array}{l}(0.71) e^{i 2 \pi(0.56)}, \\
(0.39) e^{i 2 \pi(0.43)}\end{array}\right)$ & $\left(\begin{array}{l}(0.72) e^{i 2 \pi(0.57)}, \\
(0.38) e^{i 2 \pi(0.42)}\end{array}\right)$ & $\left(\begin{array}{l}(0.73) e^{i 2 \pi(0.58)}, \\
(0.37) e^{i 2 \pi(0.41)}\end{array}\right)$ \\
\hline
\end{tabular}

\begin{tabular}{lll}
\hline Methods & Values of examined by methods & Ranking \\
\hline Garg and Rani [36] & Cannot be calculated & Cannot be calculated \\
Rani and Garg [39] & Cannot be calculated & Cannot be calculated \\
Ullah et al. [41] & $d_{\mathrm{Cq}-\mathrm{ROF}}^{\mathrm{G} 4}\left(A_{1}, B\right)=0.17$, & $A_{3} \leq A_{4} \leq A_{1} \leq A_{2}$ \\
& $d_{\mathrm{Cq}-\mathrm{ROF}}^{\mathrm{G} 4}\left(A_{2}, B\right)=0.17$, & \\
& $d_{\mathrm{Cq}-\mathrm{ROF}}^{\mathrm{G} 4}\left(A_{3}, B\right)=0.12$, & \\
& $d_{\mathrm{Cq}-\mathrm{ROF}}^{\mathrm{G} 4}\left(A_{4}, B\right)=0.14$ & \\
Proposed methods for $q=2$ & $d_{\mathrm{Cq}-\mathrm{ROF}}^{\mathrm{G} 4}\left(A_{1}, B\right)=0.16$, & \\
& $d_{\mathrm{Cq}-\mathrm{ROF}}^{\mathrm{G} 4}\left(A_{2}, B\right)=0.17$, & \\
& $d_{\mathrm{Cq}-\mathrm{ROF}}^{\mathrm{G} 4}\left(A_{3}, B\right)=0.12$, & \\
& $d_{\mathrm{Cq}-\mathrm{ROF}}^{\mathrm{G} 4}\left(A_{4}, B\right)=0.13$ & $A_{3} \leq A_{4} \leq A_{2} \leq A_{2} \leq A_{2}$ \\
Proposed methods for $q=3$ & $d_{\mathrm{Cq}-\mathrm{ROF}}^{\mathrm{G} 4}\left(A_{1}, B\right)=0.06$, & \\
& $d_{\mathrm{Cq}-\mathrm{GOF}}^{\mathrm{G} 4}\left(A_{2}, B\right)=0.08$, & \\
& $d_{\mathrm{Cq}-\mathrm{ROF}}^{\mathrm{G} 4}\left(A_{3}, B\right)=0.02$, & \\
& $d_{\mathrm{Cq}-\mathrm{ROF}}^{\mathrm{G} 4}\left(A_{4}, B\right)=0.03$ & \\
\hline
\end{tabular}


Table 15 Decision matrix for patient with respect to symptoms

Table 16 Decision matrix of complex q-rung orthopair fuzzy sets

Table 17 Comparison between proposed methods with existing methods

\begin{tabular}{lllll}
\hline Symbols & $s_{1}$ & $s_{2}$ & $s_{3}$ & $s_{4}$ \\
\hline Patient $(\mathrm{P})$ & $\left(\begin{array}{l}(0.9) e^{i 2 \pi(0.8)}, \\
(0.4) e^{i 2 \pi(0.44)}\end{array}\right)$ & $\left(\begin{array}{l}(0.92) e^{i 2 \pi(0.82)}, \\
(0.39) e^{i 2 \pi(0.43)}\end{array}\right)$ & $\left(\begin{array}{l}(0.82) e^{i 2 \pi(0.87)}, \\
(0.38) e^{i 2 \pi(0.42)}\end{array}\right)$ & $\left(\begin{array}{l}(0.83) e^{i 2 \pi(0.88)}, \\
(0.37) e^{i 2 \pi(0.41)}\end{array}\right)$ \\
\hline
\end{tabular}

\begin{tabular}{lllll}
\hline Symbols & $Q_{1}$ & $Q_{2}$ & $Q_{3}$ & $Q_{4}$ \\
\hline$s_{1}$ & $\left(\begin{array}{l}(0.91) e^{i 2 \pi(0.81)}, \\
(0.41) e^{i 2 \pi(0.41)}\end{array}\right)$ & $\left(\begin{array}{l}(0.72) e^{i 2 \pi(0.44)}, \\
(0.72) e^{i 2 \pi(0.73)}\end{array}\right)$ & $\left(\begin{array}{l}(0.82) e^{i 2 \pi(0.84)}, \\
(0.28) e^{i 2 \pi(0.4)}\end{array}\right)$ & $\left(\begin{array}{l}(0.9) e^{i 2 \pi(0.7)}, \\
(0.33) e^{i 2 \pi(0.31)}\end{array}\right)$ \\
$s_{2}$ & $\left(\begin{array}{l}(0.92) e^{i 2 \pi(0.82)}, \\
(0.42) e^{i 2 \pi(0.42)}\end{array}\right)$ & $\left(\begin{array}{l}(0.73) e^{i 2 \pi(0.46)}, \\
(0.63) e^{i 2 \pi(0.53)}\end{array}\right)$ & $\left(\begin{array}{l}(0.72) e^{i 2 \pi(0.81)}, \\
(0.18) e^{i 2 \pi(0.34)}\end{array}\right)$ & $\left(\begin{array}{l}(0.8) e^{i 2 \pi(0.8)}, \\
(0.35) e^{i 2 \pi(0.4)}\end{array}\right)$ \\
$s_{3}$ & $\left(\begin{array}{l}(0.93) e^{i 2 \pi(0.83)}, \\
(0.43) e^{i 2 \pi(0.43)}\end{array}\right)$ & $\left(\begin{array}{l}(0.74) e^{i 2 \pi(0.46)}, \\
(0.84) e^{i 2 \pi(0.73)}\end{array}\right)$ & $\left(\begin{array}{l}(0.62) e^{i 2 \pi(0.84)}, \\
(0.18) e^{i 2 \pi(0.45)}\end{array}\right)$ & $\left(\begin{array}{l}(0.85) e^{i 2 \pi(0.85)}, \\
(0.32) e^{i 2 \pi(0.21)}\end{array}\right)$ \\
$s_{4}$ & $\left(\begin{array}{l}(0.94) e^{i 2 \pi(0.84)}, \\
(0.44) e^{i 2 \pi(0.44)}\end{array}\right)$ & $\left(\begin{array}{l}(0.75) e^{i 2 \pi(0.26)}, \\
(0.75) e^{i 2 \pi(0.73)}\end{array}\right)$ & $\left(\begin{array}{l}(0.87) e^{i 2 \pi(0.83)}, \\
(0.33) e^{i 2 \pi(0.32)}\end{array}\right)$ & $\left(\begin{array}{l}(0.7) e^{i 2 \pi(0.9)}, \\
(0.34) e^{i 2 \pi(0.39)}\end{array}\right)$ \\
\hline
\end{tabular}

\begin{tabular}{lll}
\hline Methods & Values of examined by methods & Ranking \\
\hline Garg and Rani [36] & Cannot be calculated & Cannot be calculated \\
Rani and Garg [39] & Cannot be calculated & Cannot be calculated \\
Ullah et al. [41] & Cannot be calculated & Cannot be calculated \\
Proposed methods for $q=5$ & $d_{\mathrm{Cq}-\mathrm{ROF}}^{\mathrm{wG} 4}\left(Q_{1}, P\right)=0.04$, & $Q_{2} \leq Q_{3} \leq Q_{4} \leq Q_{1}$ \\
& $d_{\mathrm{Cq}-\mathrm{ROF}}^{\mathrm{wG} 4}\left(Q_{2}, P\right)=0.01$, & \\
& $d_{\mathrm{Cq}-\mathrm{ROF}}^{\mathrm{wG}}\left(Q_{3}, P\right)=0.02$, & \\
& $d_{\mathrm{Cq}-\mathrm{ROF}}^{\mathrm{wG} 4}\left(Q_{4}, P\right)=0.03$ & \\
&
\end{tabular}

Example 6 Consider the four known patterns, which are denoted the Cq-ROFNs are described in Table 18.

Suppose the unknown pattern is represented by the CqROFNs in Table 19.

Next, we will examine similarity between unknown pattern $B$ with respect to known patterns $A_{i}$ by using Eq. (46) we get.

$d_{\mathrm{Cq}-\mathrm{ROF}}^{\mathrm{G} 4}\left(A_{1}, B\right)=0.042, \quad d_{\mathrm{Cq}-\mathrm{ROF}}^{\mathrm{G} 4}\left(A_{2}, B\right)=0.013$,

$d_{\mathrm{Cq}-\mathrm{ROF}}^{\mathrm{G} 4}\left(A_{3}, B\right)=0.022, \quad d_{\mathrm{Cq}-\mathrm{ROF}}^{\mathrm{G} 4}\left(A_{4}, B\right)=0.032$

The ranking of the values is follow as:
$A_{2} \leq A_{3} \leq A_{4} \leq A_{1}$

Thus, on the based on ranking methods, we conclude that the Unknown patterns $P$ belong to the pattern $A_{i}$. In order to examine the effectiveness and dominance of the investigated approach based on dice similarity measures, we conducted a comparison between proposed approach and existing methods proposed by Garg and Rani [36], Rani and Garg [39] and Ullah et al. [41]. For this, we used the proposed approach in Eq. (46) and their corresponding results are described in Table 20, which express the unknown pattern $P$ belong to the pattern $A_{i}$.
Table 18 Decision matrix for known patterns

\begin{tabular}{lllll}
\hline Symbols & $A_{1}$ & $A_{2}$ & $A_{3}$ & $A_{4}$ \\
\hline$x_{1}$ & $\left(\begin{array}{l}(0.91) e^{i 2 \pi(0.81)}, \\
(0.41) e^{i 2 \pi(0.41)}\end{array}\right)$ & $\left(\begin{array}{l}(0.72) e^{i 2 \pi(0.44)}, \\
(0.72) e^{i 2 \pi(0.73)}\end{array}\right)$ & $\left(\begin{array}{l}(0.82) e^{i 2 \pi(0.84)} \\
(0.28) e^{i 2 \pi(0.4)}\end{array}\right)$ & $\left(\begin{array}{l}(0.9) e^{i 2 \pi(0.7)}, \\
(0.33) e^{i 2 \pi(0.31)}\end{array}\right)$ \\
$x_{2}$ & $\left(\begin{array}{l}(0.92) e^{i 2 \pi(0.82)}, \\
(0.42) e^{i 2 \pi(0.42)}\end{array}\right)$ & $\left(\begin{array}{l}(0.73) e^{i 2 \pi(0.46)}, \\
(0.63) e^{i 2 \pi(0.53)}\end{array}\right)$ & $\left(\begin{array}{l}(0.72) e^{i 2 \pi(0.81)}, \\
(0.18) e^{i 2 \pi(0.34)}\end{array}\right)$ & $\left(\begin{array}{l}(0.8) e^{i 2 \pi(0.8)}, \\
(0.35) e^{i 2 \pi(0.4)}\end{array}\right)$ \\
$x_{3}$ & $\left(\begin{array}{l}(0.93) e^{i 2 \pi(0.83)}, \\
(0.43) e^{i 2 \pi(0.43)}\end{array}\right)$ & $\left(\begin{array}{l}(0.74) e^{i 2 \pi(0.46)}, \\
(0.84) e^{i 2 \pi(0.73)}\end{array}\right)$ & $\left(\begin{array}{l}(0.62) e^{i 2 \pi(0.84)}, \\
(0.18) e^{i 2 \pi(0.45)}\end{array}\right)$ & $\left(\begin{array}{l}(0.85) e^{i 2 \pi(0.85)}, \\
(0.32) e^{i 2 \pi(0.21)}\end{array}\right)$ \\
$x_{4}$ & $\left(\begin{array}{l}(0.94) e^{i 2 \pi(0.84)}, \\
(0.44) e^{i 2 \pi(0.44)}\end{array}\right)$ & $\left(\begin{array}{l}(0.75) e^{i 2 \pi(0.26)}, \\
(0.75) e^{i 2 \pi(0.73)}\end{array}\right)$ & $\left(\begin{array}{l}(0.87) e^{i 2 \pi(0.83)}, \\
(0.33) e^{i 2 \pi(0.32)}\end{array}\right)$ & $\left(\begin{array}{l}(0.7) e^{i 2 \pi(0.9)}, \\
(0.34) e^{i 2 \pi(0.39)}\end{array}\right)$ \\
\hline
\end{tabular}


Table 19 Decision matrix for unknown patterns

\begin{tabular}{lllll}
\hline Symbols & $x_{1}$ & $x_{2}$ & $x_{3}$ & $x_{4}$ \\
\hline$B$ & $\left(\begin{array}{l}(0.9) e^{i 2 \pi(0.8)}, \\
(0.4) e^{i 2 \pi(0.44)}\end{array}\right)$ & $\left(\begin{array}{l}(0.92) e^{i 2 \pi(0.82)}, \\
(0.39) e^{i 2 \pi(0.43)}\end{array}\right)$ & $\left(\begin{array}{l}(0.82) e^{i 2 \pi(0.87)}, \\
(0.38) e^{i 2 \pi(0.42)}\end{array}\right)$ & $\left(\begin{array}{l}(0.83) e^{i 2 \pi(0.88)}, \\
(0.37) e^{i 2 \pi(0.41)}\end{array}\right)$ \\
\hline
\end{tabular}

\begin{tabular}{lll}
\hline Methods & Values of examined by methods & Ranking \\
\hline Garg and Rani [36] & Cannot be calculated & Cannot be calculated \\
Rani and Garg [39] & Cannot be calculated & Cannot be calculated \\
Ullah et al. [41] & Cannot be calculated & Cannot be calculated \\
Proposed methods for $q=5$ & $d_{\mathrm{Cq}-\mathrm{ROF}}^{\mathrm{G} 4}\left(A_{1}, B\right)=0.042$, & $A_{2} \leq A_{3} \leq A_{4} \leq A_{1}$ \\
& $d_{\mathrm{Cq}-\mathrm{ROF}}^{\mathrm{G} 4}\left(A_{2}, B\right)=0.013$, & \\
& $d_{\mathrm{Cq}-\mathrm{ROF}}^{\mathrm{G}}\left(A_{3}, B\right)=0.022$, & \\
& $d_{\mathrm{Cq}-\mathrm{ROF}}^{\mathrm{G}}\left(A_{4}, B\right)=0.032$ & \\
&
\end{tabular}

Table 20 Comparison between proposed methods with existing methods

\section{Advantages of the proposed measure}

According to the above comparison analysis, the proposed method for addressing decision-making problems has the following advantages:

1. The dice similarity measure under the CIFS [34] environment can only handle situations in which the degree of complex-valued membership and complex-valued nonmembership is provided to the decision-maker. The constraint of CIFS is that the sum of real part (Also for Imaginary Part) of membership and real part (Also for Imaginary Part) of non-membership grades are bounded to $[0,1]$. This kind of measure is unable to deal with such kind of information, whose sum is greater than 1 , which commonly occurs in real-life applications. Because CqROFSs are a successful tool to handle such kinds of information, which cannot deal effectively by CIFS and CPFS, the proposed dice similarity measure in the CqROFS can effectively be used in many real applications in decision-making.

2. The dice similarity measure under the CPFS [41] environment can only handle situations in which the degree of complex-valued membership and complex-valued nonmembership is provided to the decision-maker. The constraint of CPFS is that the sum of the square of real part (also for Imaginary Part) of membership and the square of real part (also for Imaginary Part) of nonmembership grades are bounded to $[0,1]$. This kind of measure is unable to deal with such kind of information, whose sum of the square is greater than 1 , which commonly occurs in real-life applications. Because CqROFSs are a successful tool to handle such kinds of information, which cannot deal effectively by CIFS and $\mathrm{CPFS}$, the proposed dice similarity measure in the Cq-
ROFS can effectively be used in many real applications in decision-making.

3. As has been observed from existing studies, various existing measures under CIFS and CPFS environment have been proposed by researchers, but there are some situations that cannot be distinguished by these existing measures; hence their corresponding algorithm may give an irrelevant result. The proposed measure has the ability to overcome these flaws; thus it is a more suitable measure to tackle problems.

\section{Conclusion}

The primary contribution of the work is summarized as follows:

1. In the present work, a concept of the complex q-rung orthopair fuzzy set (Cq-ROFS) has been utilized to represent the uncertain information. The Cq-ROFS is an extension of the CIFS and CPFS with a wider range of the information. The Cq-ROFS is characterized by two functions expressing the degree of complex-valued membership and degree of complex-valued non-membership. In Cq-ROFS, a flexible parameter $q$ will adjust their values to represent the information in a wider domain. Moreover, it has been concluded that the existing CIFS and CPFS are taken as a special cases of the Cq-ROFS by setting $q=1$ and $q=2$ respectively. Also, the information are represented over a domain of unit-disc complex plane.

2. In this article, taking the advantages of Cq-ROFS, we propose a novel concept of the dice similarity measures between the pairs of the different Cq-ROFSs. Several properties related to them are derived to show its existence. Further, we extend the proposed dice measures 
to its generalized dice similarity measures by introducing an attitude parameter of the decision maker $Y \in[0,1]$. Based on the decision maker behavior towards the collective information, an optimism or pessimism, a person can select their respective measurement values.

3. We define some weighted dice similarity measures and weighted generalized dice similarity measures for $\mathrm{Cq}-$ ROFS by considering the weight information to each attribute. Few properties and their axioms are derived. From these proposed measures, it is concluded that the several existing measures can be deduce from it by setting the different value of the parameter $q$ and $Y \in[0,1]$.

4. Numerical examples related to the pattern recognition and medical diagnoses are solved to explain the working of these proposed dice similarity measures. The computed results are compared with the existing studies [36, $39,41]$. Further, the advantages of the proposed measures over these existing measures are illustrated with some numerical examples (in Examples 3-5). It is obtained from them that the under some certain cases, the existing approached defined in $[36,39,41]$ are unable to handle the information and hence does not solve the decision making problems, while the proposed measures have successfully solve each of them.

In the future, we will extend the current study to the different applications such as image processing, decisionmaking problems etc. Also, there is a scope of elongating the presented work by considering the information about the parameter in the soft set theory [46-50].

\section{Compliance with ethical standards}

\section{Conflict of interest There is no conflict of interest.}

Open Access This article is licensed under a Creative Commons Attribution 4.0 International License, which permits use, sharing, adaptation, distribution and reproduction in any medium or format, as long as you give appropriate credit to the original author(s) and the source, provide a link to the Creative Commons licence, and indicate if changes were made. The images or other third party material in this article are included in the article's Creative Commons licence, unless indicated otherwise in a credit line to the material. If material is not included in the article's Creative Commons licence and your intended use is not permitted by statutory regulation or exceeds the permitted use, you will need to obtain permission directly from the copyright holder. To view a copy of this licence, visit http://creativecomm ons.org/licenses/by/4.0/.

\section{References}

1. Zadeh LA (1965) Fuzzy sets. Inf Control 8:338-353
2. Zimmermann HJ (2001) Fuzzy set theory and its applications. Kluwer Academic Publishers, London

3. Steimann F (1997) Fuzzy set theory in medicine. Artif Intell Med 11(1):1-7

4. Atanassov KT (1986) Intuitionistic fuzzy sets. Fuzzy Sets Syst 20(1):87-96

5. Dengfeng L, Chuntian C (2002) New similarity measure of intuitionistic fuzzy sets and application to pattern recognitions. Pattern Recogn Lett 23:221-225

6. Chen SM, Cheng SH, Lan TC (2016) A novel similarity measure between intuitionistic fuzzy sets based on the centroid points of transformed fuzzy numbers with applications to pattern recognition. Inf Sci 343-344:15-40

7. De SK, Biswas R, Roy A (2001) An application of intuitionistic fuzzy sets in medical diagnosis. Fuzzy Sets Syst 117:209-213

8. Szmidt E, Kacprzyk J (2004) A similarity measure for intuitionistic fuzzy sets and its application in supporting medical diagnostic reasoning. Lect Notes Comput Sci 3070:388-393

9. Liu P (2014) Some hamacher aggregation operators based on the interval-valued intuitionistic fuzzy numbers and their application to group decision making. IEEE Trans Fuzzy Syst 22(1):83-97

10. Hung WL, Yang MS (2004) Similarity measures of intuitionistic fuzzy sets based on hausdorff distance. Pattern Recogn Lett 25:1603-1611

11. Xu ZS, Chen J (2008) "An overview of distance and similarity of intuitionistic fuzzy sets", International Journal of Uncertainty, Fuzziness. Knowl-Based Syst 16:529-555

12. Yager RR (2013) Pythagorean fuzzy subsets. In: Procedings Joint IFSA World Congress and NAFIPS Annual Meeting, Edmonton, Canada, pp 57-61

13. Garg H (2016) A novel correlation coefficients between Pythagorean fuzzy sets and its applications to decision-making processes. Int J Intell Syst 31(12):1234-1252

14. Peng X, Yang Y (2015) Some results for Pythagorean fuzzy sets. Int J Intell Syst 30(11):1133-1160

15. Zhang XL, Xu ZS (2014) Extension of TOPSIS to multi-criteria decision making with Pythagorean fuzzy sets. Int J Intell Syst 29(12):1061-1078

16. Garg H (2016) A new generalized Pythagorean fuzzy information aggregation using Einstein operations and its application to decision making. Int J Intell Syst 31(9):886-920

17. Garg H (2017) Generalized Pythagorean fuzzy geometric aggregation operators using Einstein t-norm and t-conorm for multicriteria decision-making process. Int J Intell Syst 32(6):597-630

18. Peng X, Garg H (2019) Multiparametric similarity measures on Pythagorean fuzzy sets with applications to pattern recognition. Appl Intell 49(12):4058-4096

19. Wang L, Li N (2020) Pythagorean fuzzy interaction power Bonferroni mean aggregation operators in multiple attribute decision making. Int J Intell Syst 35(1):150-183

20. Garg H (2020) Neutrality operations-based pythagorean fuzzy aggregation operators and its applications to multiple attribute group decision-making process. J Ambient Intell Hum Comput 11(7):3021-3041

21. Garg H (2019) Novel neutrality operation-based pythagorean fuzzy geometric aggregation operators for multiple attribute group decision analysis. Int J Intell Syst 34(10):2459-2489

22. Peng X, Selvachandran G (2019) Pythagorean fuzzy set: state of the art and future directions. Artif Intell Rev 52:1873-1927. https:// doi.org/10.1007/s10462-017-9596-9

23. Yager RR (2017) Generalized orthopair fuzzy sets. IEEE Trans Fuzzy Syst 25(5):1222-1230

24. Liu P, Wang P (2018) Some q-rung orthopair fuzzy aggregation operators and their applications to multiple-attribute decision making. Int J Intell Syst 33(2):259-280 
25. G. Wei, H. Gao, and Y. Wei (2018) Some q-rung orthopair fuzzy Heronian mean operators in multiple attribute decision making. Int J Intell Syst 33(7):1426-1458. https://doi.org/10.1002/int.21985

26. Liu P, Wang P (2019) Multiple-attribute decision-making based on Archimedean Bonferroni operators of q-rung orthopair fuzzy numbers. IEEE Trans Fuzzy Syst 27(5):834-848

27. Garg H, Chen S-M (2020) Multiattribute group decision making based on neutrality aggregation operators of q-rung orthopair fuzzy sets. Inf Sci 517:427-447

28. Liu P, Liu J (2018) Some q-rung orthopair fuzzy bonferroni mean operators and their application to multi-attribute group decision making. Int J Intell Syst 33(2):315-347

29. Peng X, Dai J, Garg H (2018) Exponential operation and aggregation operator for q-rung orthopair fuzzy set and their decisionmaking method with a new score function. Int J Intell Syst 33(11):2255-2282

30. Xing Y, Zhang R, Zhou Z, Wang J (2019) Some q-rung orthopair fuzzy point weighted aggregation operators for multi-attribute decision making. Soft Comput 23:11627-11649

31. Garg H (2020) A novel trigonometric operation-based q-rung orthopair fuzzy aggregation operators and its fundamental properties. Neural Comput Appl 32:15077-15099. https://doi.org/10.1 007/s00521-020-04859-x

32. Ramot D, Milo R, Fiedman M, Kandel A (2002) Complex fuzzy sets. IEEE Trans Fuzzy Syst 10(2):171-186

33. Ramot D, Friedman M, Langholz G, Kandel A (2003) Complex fuzzy logic. IEEE Trans Fuzzy Syst 11(4):450-461

34. Alkouri A, Salleh A (2012) Complex intuitionistic fuzzy sets, 2012, vol. 1482, ch. In: 2nd International conference on fundamental and applied sciences, pp 464-470

35. Kumar T, Bajaj RK (2014) On complex intuitionistic fuzzy soft sets with distance measures and entropies. J Math 2014:12

36. Garg H, Rani D (2019) A robust correlation coefficient measure of complex intuitionistic fuzzy sets and their applications in decisionmaking. Appl Intell 49(2):496-512

37. Garg H, Rani D (2019) Some generalized complex intuitionistic fuzzy aggregation operators and their application to multicriteria decision-making process. Arab J Sci Eng 44(3):2679-2698

38. Rani D, Garg H (2017) Distance measures between the complex intuitionistic fuzzy sets and its applications to the decision-making process. Int J Uncertain Quantif 7(5):423-439
39. Rani D, Garg H (2018) Complex intuitionistic fuzzy power aggregation operators and their applications in multi-criteria decisionmaking. Expert Syst 35(6):e12325

40. Garg H, Rani D (2019) Complex interval- valued intuitionistic fuzzy sets and their aggregation operators. Fund Inf 164(1):61-101

41. Ullah K, Mahmood T, Ali Z, Jan N (2020) On some distance measures of complex Pythagorean fuzzy sets and their applications in pattern recognition. Complex Intell Syst 6(1):15-27. 10.1007/s40747-019-0103-6

42. Akram M, Garg H, Zahid K (2020) Extensions of ELECTRE-I and TOPSIS methods for group decision-making under complex Pythagorean fuzzy environment. Iran J Fuzzy Syst 17(5):147-164

43. Liu P, Mahmood T, Ali Z (2020) Complex q-rung orthopair fuzzy aggregation operators and their applications in multi-attribute group decision making. Information 11(1):5

44. Garg H, Gwak J, Mahmood T, Ali Z (2020) Power aggregation operators and VIKOR methods for complex q-rung orthopair fuzzy sets and their applications. Mathematics 8(4):538. https://doi.org/ $10.3390 /$ math 8040538

45. Dice LR (1945) Measures of the amount of ecologic association between species. Ecology 26(3):297-302

46. Garg H, Arora R (2020) TOPSIS method based on correlation coefficient for solving decision-making problems with intuitionistic fuzzy soft set information. AIMS Math 5(4):2944-2966

47. Petchimuthu S, Garg H, Kamaci H, Atagün AO (2020) The mean operators and generalized products of fuzzy soft matrices and their applications in mcgdm. Comput Appl Math 39(2):1-32. 10.1007/s40314-020-1083-2

48. Garg H, Arora R (2018) Generalized and group-based generalized intuitionistic fuzzy soft sets with applications in decision-making. Appl Intell 48(2):343-356

49. Kamaci H (2019) Similarity measure for soft matrices and its application. J Intell Fuzzy Syst 36(4):3061-3072

50. Kamaci H (2020) Selectivity analysis of parameters in soft set and its effect on decision making. Int J Mach Learn Cybern 11:313-324

Publisher's Note Springer Nature remains neutral with regard to jurisdictional claims in published maps and institutional affiliations. 